\title{
Equivariant cohomology and equivariant intersection theory
}

\author{
Michel Brion
}

February 1, 2008

\begin{abstract}
This text is an introduction to equivariant cohomology, a classical tool for topological transformation groups, and to equivariant intersection theory, a much more recent topic initiated by D. Edidin and W. Graham. It is based on lectures given at Montréal is Summer 1997.

Our main aim is to obtain explicit descriptions of cohomology or Chow rings of certain manifolds with group actions which arise in representation theory, e.g. homogeneous spaces and their compactifications.

As another appplication of equivariant intersection theory, we obtain simple versions of criteria for smoothness or rational smoothness of Schubert varieties (due to Kumar [40], Carrell-Peterson [16] and Arabia [4]) whose statements and proofs become quite transparent in this framework.

We now describe in more detail the contents of these notes; the prerequisites are notions of algebraic topology, compact Lie groups and linear algebraic groups. Sections 1 and 2 are concerned with actions of compact Lie groups on topological spaces, especially on symplectic manifolds. The material of Section 1 is classical: universal bundles, equivariant cohomology and its relation to usual cohomology, and the localization theorem for actions of compact tori.

A useful refinement of the latter theorem is presented in Section 2, based on joint work with Michèle Vergne. It leads to a complete description of the cohomology ring of compact multiplicity-free spaces. Examples include coadjoint orbits, projective toric manifolds and De Concini-Procesi's complete symmetric varieties 18 .

The subject of the last three sections is equivariant intersection theory for actions of linear algebraic groups on schemes. Edidin and Graham's equiv-
\end{abstract}


ariant Chow groups are introduced in Section 3, after a brief discussion of usual Chow groups. The basic properties of these equivariant groups are presented, as well as their applications to Chow groups of quotients (after [22]) and their relation to usual Chow groups (after [14]). As a direct application, we obtain a structure theorem for the rational Chow ring of a homogeneous space under a linear algebraic group; it turns out to be much simpler than the corresponding rational cohomology ring.

Section 4, based on [14], presents Edidin and Graham's localization theorem concerning equivariant Chow groups for torus actions, and its refined version for projective smooth varieties. Then we introduce equivariant multiplicities at isolated fixed points, we relate this notion to work of Joseph and Rossmann [34], [45] in representation theory, and we determine equivariant multiplicities of Schubert varieties.

This is applied in Section 5 to criteria for smoothness or rational smoothness of Schubert varieties, in terms of their equivariant multiplicities. We deduce these criteria from new, more general results concerning (rational) smoothness at an "attractive" fixed point of a torus action; these results are closely related to recent work of Arabia [4].

The exposition follows the original five lectures, except in Section 5 which replaces a lecture on operators of divided differences, based on [14]. Indeed, we chose to develop here the applications of equivariant intersection theory to singularities of Schubert varieties, thereby answering questions raised by several participants of the summer school. Further applications to spherical varieties will be given elsewhere.

\section{Equivariant cohomology}

Let $X$ be a topological space and let $k$ be a commutative ring. We denote by $H^{*}(X, k)$ the cohomology ring of $X$ with coefficients in $k$. We will be mainly interested in the case where $k=\mathbb{Q}$ is the field of rational numbers; we set $H^{*}(X):=H^{*}(X, \mathbb{Q})$.

Recall that $H^{*}(X, k)$ is a graded $k$-algebra, depending on $X$ in a contravariant way: Any continuous map $f: X \rightarrow Y$ to a topological space $Y$ induces a $k$-algebra homomorphism of degree zero $f^{*}: H^{*}(Y, k) \rightarrow H^{*}(X, k)$ which only depends on the homotopy class of $f$.

Consider now a topological space $X$ endowed with the action of a topological group $G$; we will say that $X$ is a $G$-space. There exists a principal 
$G$-bundle

$$
p: E_{G} \rightarrow B_{G}
$$

such that the space $E_{G}$ is contractible, and such a bundle is universal among principal $G$-bundles (see e.g. [32] Chapter IV; a construction of universal bundles will be given below when $G$ is a compact Lie group). The group $G$ acts diagonally on $X \times E_{G}$ and the quotient

$$
X \times E_{G} \rightarrow\left(X \times E_{G}\right) / G=: X \times_{G} E_{G}
$$

exists. Define the equivariant cohomology ring $H_{G}^{*}(X, k)$ by

$$
H_{G}^{*}(X, k):=H^{*}\left(X \times_{G} E_{G}, k\right) .
$$

In particular, for $X$ a point, we have

$$
H_{G}^{*}(p t, k)=H^{*}\left(E_{G} / G, k\right)=H^{*}\left(B_{G}, k\right) .
$$

Because $p: E_{G} \rightarrow B_{G}$ is a principal bundle, the projection

$$
p_{X}: X \times_{G} E_{G} \rightarrow E_{G} / G=B_{G}
$$

is a fibration with fiber $X$. Thus, $H_{G}^{*}(X, k)$ is an algebra over $H_{G}^{*}(p t, k)$. Moreover, restriction to the fiber of $p_{X}$ defines an algebra homomorphism

$$
\rho: H_{G}^{*}(X, k) /\left(H_{G}^{+}(p t, k)\right)=H_{G}^{*}(X, k) \otimes_{H_{G}^{*}(p t, k)} k \rightarrow H^{*}(X, k)
$$

where $\left(H_{G}^{+}(p t, k)\right)$ denotes the ideal of $H_{G}^{*}(X, k)$ generated by images of homogeneous elements of $H_{G}^{*}(p t, k)$ of positive degree.

We will see that, for certain spaces $X$ and for rational coefficients, the map $\rho$ is an isomorphism, and that the equivariant cohomology algebra $H_{G}^{*}(X)$ can be described completely; as a consequence, we will obtain a description of the usual cohomology algebra of $X$.

But it may happen that the map $\rho$ is trivial. Consider for example a compact connected Lie group $G$ acting on $X=G$ by left multiplication. Then

$$
H_{G}^{*}(X)=H^{*}\left(\left(G \times E_{G}\right) / G\right)=H^{*}\left(E_{G}\right)=\mathbb{Q}
$$

whereas $H^{*}(X)=H^{*}(G)$ is an exterior algebra on $l$ generators, where $l$ is the rank of $G$ (a classical theorem of Hopf [30]). 


\section{Remarks}

1) The equivariant cohomology ring is independent of the choice of a universal bundle. Indeed, if $E_{G}^{\prime} \rightarrow B_{G}^{\prime}$ is another such bundle, then the projections $\left(X \times E_{G} \times E_{G}^{\prime}\right) / G \rightarrow X \times{ }_{G} E_{G}$ and $\left(X \times E_{G} \times E_{G}^{\prime}\right) / G \rightarrow X \times{ }_{G} E_{G}^{\prime}$ are fibrations with contractible fibers $E_{G}^{\prime}$ and $E_{G}$. Thus, both projections induce isomorphisms in cohomology.

2) If $G$ acts trivially on $X$, then $X \times_{G} E_{G}=X \times B_{G}$. By the Künneth isomorphism, it follows that

$$
H_{G}^{*}(X) \simeq H_{G}^{*}(p t) \otimes H^{*}(X) .
$$

In particular, the $H_{G}^{*}(p t)$-module $H_{G}^{*}(X)$ is free.

On the other hand, if $G$ acts on $X$ with a quotient space $X / G$ and with finite isotropy groups, then

$$
H_{G}^{*}(X) \simeq H^{*}(X / G)
$$

Indeed, the quotient map induces a map

$$
\pi: X \times_{G} E_{G} \rightarrow X / G
$$

whose fibers are the quotients $E_{G} / G_{x}$ where $G_{x}$ denotes the isotropy group of $x \in X$. Because $G_{x}$ is finite and $E_{G}$ is contractible, the fibers of $\pi$ are Q-acyclic. Thus, $\pi^{*}: H^{*}(X / G) \rightarrow H_{G}^{*}(X)$ is an isomorphism.

3 ) Let $H$ be a closed subgroup of $G$. Then the quotient $E_{G} / H$ exists and the map $E_{G} \rightarrow E_{G} / H$ is a universal bundle for $H$. As a consequence, we obtain the following description of the $G$-equivariant cohomology ring of the homogeneous space $G / H$ :

$$
H_{G}^{*}(G / H, k) \simeq H_{H}^{*}(p t, k)
$$

Indeed, we have

$$
H_{G}^{*}(G / H, k)=H^{*}\left(G / H \times_{G} E_{G}, k\right)=H^{*}\left(E_{G} / H, k\right) .
$$

More generally, let $Y$ be a $H$-space. The quotient of $G \times Y$ by the diagonal action of $H$ exists; we denote it by $G \times_{H} Y$. Then we obtain as above:

$$
H_{G}^{*}\left(G \times_{H} Y, k\right) \simeq H_{H}^{*}(Y, k)
$$


4) Let $n$ be a positive integer. Let $E_{G}^{n} \rightarrow B_{G}^{n}$ be a principal $G$-bundle such that $E_{G}^{n}$ is $n$-connected (that is, any continuous map from a sphere of dimension at most $n$ to $E_{G}^{n}$ is homotopic to the constant map). Then

$$
H_{G}^{m}(X, k)=H^{m}\left(X \times_{G} E_{G}^{n}, k\right)
$$

for any compact topological $G$-manifold $X$ of dimension at most $n$, and for any integer $m \leq n$ [32] Chapter IV, Theorem 13.1. In other words, we can compute any homogeneous component of $H_{G}^{*}(X)$ by finite approximations of $E_{G}$. This will be the starting point for the definition of equivariant Chow groups, in Section 3.

\section{Examples}

1) $G=S^{1}$ (the multiplicative group of complex numbers of modulus one). Let $G$ act on $\mathbb{C}^{n}$ by scalar multiplication; then the unit sphere $S^{2 n-1}$ is $G$ stable and the quotient $S^{2 n-1} / G$ is complex projective space $\mathbb{P}^{n-1}$. We thus obtain a principal bundle $S^{2 n-1} \rightarrow \mathbb{P}^{n-1}$ with total space $(2 n-2)$-connected. So we can take $E_{G}=\lim _{\rightarrow} S^{2 n-1}=S^{\infty}$; then $B_{G}=\lim _{\rightarrow} \mathbb{P}^{n}=\mathbb{P}^{\infty}$. It follows that

$$
H_{G}^{*}(p t, k)=H^{*}\left(B_{G}, k\right)=k[t]
$$

where $t$ is an indeterminate of degree 2 .

2) More generally, let $G$ be a (compact) torus. Then $G \simeq\left(S^{1}\right)^{\ell}$ and thus, we can take $E_{G}=\left(E_{S^{1}}\right)^{\ell}$. In particular, $H_{G}^{*}(p t, k)$ is a polynomial $k$-algebra on $\ell$ indeterminates of degree 2. A more intrinsic description of $H_{G}^{*}(p t)$ is as follows. Denote by $\Xi(G)$ the character group of $G$ consisting of all continuous group homomorphisms $G \rightarrow S^{1}$. Any $\chi \in \Xi(G)$ defines a one-dimensional complex representation of $G$ with space $\mathbb{C} \chi$. Consider the associated complex line bundle

$$
L(\chi):=\left(E_{G} \times_{G} \mathbb{C} \chi \rightarrow B_{G}\right)
$$

and its first Chern class $c(\chi) \in H^{2}\left(B_{G}\right)$. Let $S$ be the symmetric algebra over $\mathbb{Q}$ of the group $\Xi(G)$. Then $S$ is a polynomial ring on $\ell$ generators of degree 1 , and the map $\chi \rightarrow c(\chi)$ extends to a ring isomorphism

$$
c: S \rightarrow H_{G}^{*}(p t)
$$

which doubles degrees: the characteristic homomorphism.

3) Finally, let $G$ be a compact Lie group. Then we can embed $G$ as a closed subgroup of some $G L_{n}$, where $G L_{n}$ denotes the group of invertible 
$n \times n$ complex matrices. We now construct a universal bundle $E_{G L_{n}} \rightarrow B_{G L_{n}}$ for the group $G L_{n}$; then $E_{G L_{n}} \rightarrow E_{G L_{n}} / G$ will be a universal bundle for $G$.

Let $N \geq n$ be an integer. Consider the space $M_{N \times n}$ of all $N \times n$ complex matrices, and its subset $M_{N \times n}^{\max }$ of matrices of maximal rank $n$. Clearly, $G L_{n}$ acts on $M_{N \times n}$ and preserves $M_{N \times n}^{\max }$. Let $G r_{N, n}$ be the Grassmann variety of linear subspaces of dimension $n$ of $\mathbb{C}^{N}$. With any matrix $A \in M_{N \times n}^{\max }$, we associate its image $\operatorname{Im}(A)$. This defines a map

$$
M_{N \times n}^{\max } \rightarrow G r_{N, n}
$$

which is a principal $G L_{n}$-bundle. Moreover, the complement of $M_{N \times n}^{\max }$ in $M_{N \times n}$ is a (Zariski) closed subset of $M_{N \times n}$ of codimension $N-n+1$ over $\mathbb{C}$. It follows that $M_{N \times n}^{\max }$ is $(N-n)$-connected. So we obtain a construction of $E_{G L_{n}}$ as the space of $\infty \times n$ complex matrices of maximal rank.

We now show that equivariant cohomology for a compact connected Lie group can be described in terms of equivariant cohomology for a maximal torus.

Proposition 1 Let $G$ be a compact connected Lie group and let $T \subset G$ be a maximal torus with normalizer $N$ and with Weyl group $W=N / T$; let $X$ be a G-space.

(i) The group $W$ acts on $H_{T}^{*}(X)$ and we have an isomorphism

$$
H_{G}^{*}(X) \simeq H_{T}^{*}(X)^{W}
$$

In particular, $H_{G}^{*}(p t)$ is isomorphic to $S^{W}$ where $S$ denotes the symmetric algebra of the character group $\Xi(T)$ (occuring in degree 2), and $S^{W}$ the ring of $W$-invariants in $S$.

(ii) The map

$$
S \simeq H_{G}^{*}(G / T) \rightarrow H^{*}(G / T)
$$

is surjective and induces an isomorphism $S /\left(S_{+}^{W}\right) \rightarrow H^{*}(G / T)$ where $\left(S_{+}^{W}\right)$ denotes the ideal of $S$ generated by all homogeneous $W$-invariants of positive degree.

(iii) We have an isomorphism

$$
S \otimes_{S^{W}} H_{G}^{*}(X) \simeq H_{T}^{*}(X) .
$$

In particular, $H_{T}^{*}(G / T)$ is isomorphic to $S \otimes_{S^{W}} S$. 
Proof (after [31] Chapter III, §1).

(i) Let $G^{\mathbb{C}}$ be the complexification of $G$; then $G^{\mathbb{C}}$ is a complex connected reductive Lie group. Let $B$ be a Borel subgroup of $G^{\mathbb{C}}$ containing the compact torus $T$. Then, by the Iwasawa decomposition, we have $G^{\mathbb{C}}=G B$ and $G \cap B=T$. Thus, the map $G / T \rightarrow G^{\mathbb{C}} / B$ is a homeomorphism. By the Bruhat decomposition, the flag manifold $G^{\mathbb{C}} / B$ has a stratification by $|W|$ strata, each of them being isomorphic to a complex affine space. It follows that $H^{*}(G / T)$ vanishes in odd degrees, and that the topological Euler characteristic $\chi(G / T)$ is equal to $|W|$.

Because the finite group $W$ acts freely on $G / T$ with quotient $G / N$, we have an isomorphism

$$
H^{*}(G / N) \simeq H^{*}(G / T)^{W}
$$

and moreover, $\chi(G / N)=|W|^{-1} \chi(G / T)=1$. It follows that $H^{*}(G / N)$ vanishes in odd degrees and is one-dimensional. In other words, $G / N$ is $\mathbb{Q}$-acyclic.

Thus, the fibration

$$
X \times_{N} E_{G} \rightarrow X \times_{G} E_{G}
$$

with fiber $G / N$ induces an isomorphism $H_{G}^{*}(X) \rightarrow H_{N}^{*}(X)$. Moreover, we have a covering

$$
X \times_{T} E_{G} \rightarrow X \times_{N} E_{G}
$$

with group $W$, whence $H_{N}^{*}(X) \simeq H_{T}^{*}(X)^{W}$.

(ii) By (i) applied to the point, the odd degree part of

$$
H^{*}\left(B_{G}\right)=H_{G}^{*}(p t)=S^{W}
$$

is zero. The fibration

$$
G / T \times_{G} E_{G} \rightarrow B_{G}
$$

has fiber $G / T$ with vanishing odd cohomology, too. Thus, the Leray spectral sequence degenerates, and we obtain an isomorphism

$$
H^{*}(G / T) \simeq H_{G}^{*}(G / T) /\left(H^{*}\left(B_{G}\right)_{+}\right)=H_{T}^{*}(p t) /\left(H_{T}^{*}(p t)_{+}^{W}\right)=S /\left(S_{+}^{W}\right) .
$$

(iii) For the fibration

$$
X \times_{T} E_{G} \rightarrow X \times_{G} E_{G}
$$


with fiber $G / T$, restriction to fiber defines a ring homomorphism $H_{T}^{*}(X) \rightarrow$ $H^{*}(G / T)$ which is surjective, because the map $S \rightarrow H^{*}(G / T)$ is. Thus, the Leray spectral sequence degenerates, and $H_{T}^{*}(X) \simeq H_{G}^{*}(X) \otimes H^{*}(G / T)$ as a $H_{G}^{*}(X)$-module. This implies our statement.

Remark. The map $S \rightarrow H^{*}(G / T)$ is the characteristic homomorphism, which associates with any $\chi \in \Xi(T)$ the Chern class of the corresponding line bundle on $G / T$. The map $S \otimes_{S^{W}} S \rightarrow H_{T}^{*}(G / T)$ is a lift of this characteristic homomorphism to $T$-equivariant cohomology.

For $G$ and $X$ as before, consider the restriction map

$$
\rho: H_{G}^{*}(X) /\left(S_{+}^{W}\right) \rightarrow H^{*}(X) .
$$

We already saw that $\rho$ may not be surjective; however, there is an important class of $G$-spaces for which $\rho$ is an isomorphism.

Definition. Let $G$ be a compact connected Lie group and let $X$ be a $G$ space. Then $X$ is a symplectic $G$-manifold if $X$ is a smooth manifold with a non-degenerate closed, $G$-invariant form $\omega$ (the symplectic form). Moreover, $X$ is a Hamiltonian $G$-space if it satisfies the following additional condition:

There exists a $G$-equivariant smooth map $\mu: X \rightarrow \mathfrak{g}^{*}$ (where $\mathfrak{g}$ denotes the Lie algebra of $G$ ) such that, for any $x \in X, \xi \in T_{x} X$ and $\eta \in \mathfrak{g}$, we have:

$$
d \mu_{x}(\xi)(\eta)=\omega_{x}\left(\xi, \eta_{x}\right)
$$

(where $\eta_{x}$ is the value at $x$ of the vector field on $X$ defined by $\eta \in \mathfrak{g}$ ).

The map $\mu$ is called a moment map for the action of $G$ on $X$; it is unique up to translation by a central element of $\mathfrak{g}$. A Hamiltonian $G$-space $X$ is also Hamiltonian for the action of any closed connected subgroup $H$ of $G$; the moment map for this action is $\mu$ followed by restriction $\mathfrak{g}^{*} \rightarrow \mathfrak{h}^{*}$.

Examples of compact Hamiltonian $G$-spaces are complex projective manifolds with a linear action of $G$. More precisely, let $V$ be the space of a finite-dimensional complex representation of $G$, let $\mathbb{P}(V)$ be its projectivization, and let $X \subset \mathbb{P}(V)$ be a $G$-invariant projective submanifold. The choice of a $G$-invariant Hermitian scalar product $(\cdot, \cdot)$ on $V$ defines a symplectic form on $\mathbb{P}(V)$ (the imaginary part of the Fubini-Study metric). This makes $\mathbb{P}(V)$ a Hamiltonian $G$-space with moment map given by

$$
\mu([v])(\eta)=\frac{(v, \eta v)}{2 i \pi(v, v)}
$$


where $[v] \in \mathbb{P}(V)$ is the image of $v \in V$, and $\eta \in \mathfrak{g}$. Moreover, $X$ is a Hamiltonian subspace of $\mathbb{P}(V)$ 38.

Now we can state the following result, due to F. Kirwan [38] Proposition 5.8 .

Proposition 2 Let $G$ be a compact connected Lie group and let $X$ be a compact Hamiltonian $G$-space. Then, notation being as above, the $S^{W}$-module $H_{G}^{*}(X)$ is free, and the map $\rho: H_{G}^{*}(X) /\left(S_{+}^{W}\right) \rightarrow H^{*}(X)$ is an isomorphism.

As an example, consider the $G$-space $X=G / T$ where, as before, $T$ is a maximal torus of $G$. Embed $G / T$ into $\mathfrak{g}^{*}$ as the orbit of a regular element. Then, as a coadjoint orbit, $G / T$ is a Hamiltonian $G$-manifold and the moment map is simply inclusion $G / T \rightarrow \mathfrak{g}^{*}$, see e.g. [5] Chapter II, 3.3. In this way, we recover the structure of the cohomology ring of the flag manifold $G / T$.

A powerful tool in equivariant cohomology is the following localization theorem of Borel-Atiyah-Segal [31] Chapter III, $§ 2$.

Theorem 3 Let $T$ be a compact torus and let $X$ be a $T$-space which embeds equivariantly into a finite-dimensional T-module. Let $i_{T}: X^{T} \rightarrow X$ be the inclusion of the fixed point set. Then the $S$-linear map

$$
i_{T}^{*}: H_{T}^{*}(X) \rightarrow H_{T}^{*}\left(X^{T}\right)
$$

becomes an isomorphism after inverting finitely many non-trivial characters.

Proof For later use, we prove the following stronger statement.

Lemma 4 Let $\Gamma \subset T$ be a closed subgroup and let $i_{\Gamma}: X^{\Gamma} \rightarrow X$ be the inclusion of the fixed point set. If $X$ embeds equivariantly into a finite-dimensional $T$-module, then the restriction map

$$
i_{\Gamma}^{*}: H_{T}^{*}(X) \rightarrow H_{T}^{*}\left(X^{\Gamma}\right)
$$

becomes an isomorphism after inverting finitely many characters of $T$ which restrict non-trivially to $\Gamma$.

Proof First we consider the case where $X^{\Gamma}$ is empty. Then we have to prove that $H_{T}^{*}(X)$ is killed by a product of characters of $T$ which restrict non-trivially to $\Gamma$. Because $X$ embeds into a finite-dimensional $T$-module, 
we can cover $X$ by finitely many $T$-invariant subsets $X_{1}, \ldots, X_{n}$ such that every $X_{j}$ admits a $T$-equivariant map $X_{j} \rightarrow T / \Gamma_{j}$ where $\Gamma_{j} \subset T$ is a closed subgroup which does not contain $\Gamma$ (exercise). Thus, we can choose $\chi_{j} \in \Xi(T)$ such that $\chi_{j}$ restricts trivially to $\Gamma_{j}$ but not to $\Gamma$. For any $T$-invariant subset $U_{j} \subset X_{j}$, the ring $H_{T}^{*}\left(U_{j}\right)$ is a module over $H_{T}^{*}\left(T / \Gamma_{j}\right)$. The image of $\chi_{j}$ in the latter is zero; thus, multiplication by $\chi_{j}$ is zero in $H_{T}^{*}\left(U_{j}\right)$. By MayerVietoris, multiplication by the product of the $\chi_{j}$ is zero in $H_{T}^{*}(X)$.

In the general case, let $Y \subset X$ be a closed $T$-stable neighborhood of $X^{\Gamma}$ in $X$. Let $Z$ be the closure of $X \backslash Y$ in $X$; then $Z$ is $T$-stable and $Z^{\Gamma}$ is empty. By the first step of the proof and Mayer-Vietoris, it follows that restriction $H_{T}^{*}(X) \rightarrow H_{T}^{*}(Y)$ is an isomorphism after inverting a finite set $\mathcal{F}$ of characters of $T$. Moreover, $\mathcal{F}$ is independent of $Y$, because it only depends on a $T$-module which contains $X$. To conclude the proof, observe that $H_{T}^{*}\left(X^{\Gamma}\right)$ is the direct limit of the $H_{T}^{*}(Y)$.

By [36] Theorem 4.12, the assumptions of Theorem 3 hold if $X$ is a compact manifold. If moreover $X$ is a symplectic $T$-manifold, then $X^{T}$ is, too, as a consequence of the following

Lemma 5 Let $G$ be a compact connected Lie group, $\Gamma \subset G$ a closed subgroup with centralizer $G^{\Gamma}$, and $X$ a symplectic $G$-manifold. Then the fixed point set $X^{\Gamma}$ is a symplectic $G^{\Gamma}$-manifold, and the normal bundle $N_{X, X^{\Gamma}}$ has a natural structure of a complex vector bundle.

If moreover the $G$-action on $X$ is Hamiltonian with moment map $\mu$, then the $G^{\Gamma}$-action on $X^{\Gamma}$ is Hamiltonian with moment map: restriction of $\mu$ followed by restriction to $\mathfrak{g}^{\Gamma}$.

Proof Let $x \in X^{\Gamma}$. Because $\Gamma$ is compact, there exists a $\Gamma$-invariant neighborhood of $x$ in $X$ which is equivariantly isomorphic to a $\Gamma$-module 36 Theorem 4.10. It follows that $X^{\Gamma}$ is a closed submanifold of $X$, and that we have the equality of tangent spaces

$$
T_{x}\left(X^{\Gamma}\right)=\left(T_{x} X\right)^{\Gamma}
$$

Moreover, $\left(T_{x} X\right)^{\Gamma}$ has a unique $\Gamma$-invariant complement in $T_{x} X$ : the span of all $\gamma \xi-\xi$ where $\gamma \in \Gamma$ and $\xi \in T_{x} X$. Because the symplectic form $\omega_{x}$ is $\Gamma$-invariant, it follows that $\left(T_{x} X\right)^{\Gamma}$ is a symplectic subspace of $T_{x} X$. Thus, restriction $\omega^{\Gamma}$ of $\omega$ to $X^{\Gamma}$ is non-degenerate, and closed because $\omega$ is. 
If moreover $X$ is Hamiltonian with moment map $\mu$, then we have for $\xi \in\left(T_{x} X\right)^{\Gamma}$ and $\eta \in \mathfrak{g}^{\Gamma}$ :

$$
\omega_{x}^{\Gamma}\left(\xi, \eta_{x}\right)=\omega_{x}\left(\xi, \eta_{x}\right)=d \mu_{x}(\xi)(\eta),
$$

that is, $\left.\mu\right|_{X^{\Gamma}}$ is a moment map for the action of $G^{\Gamma}$.

For a symplectic $T$-manifold $X$, this leads to an explicit form of the localization theorem. Let $Y$ be a connected component of $X^{T}$, then the codimension of $Y$ in $X$ is an even number, say $2 n$, and we have a Gysin map

$$
i_{Y *}: H_{T}^{*}(Y) \rightarrow H_{T}^{*}(X)
$$

which increases degree by $2 n$. Composition $i_{Y}^{*} \circ i_{Y *}$ is multiplication by the equivariant Euler class of $Y$ in $X$, which we denote by $E u_{T}(Y, X)$ : because the normal bundle $N_{Y, X}$ is a complex equivariant vector bundle, it admits equivariant Chern classes in $H_{T}^{*}(Y)$ (which are the Chern classes of the vector bundle $\left(Y \times N_{Y, X} \times E_{T}\right) / T$ over $\left.Y_{T}\right)$ and $E u_{T}(Y, X)$ is the top equivariant Chern class $c_{n, T}\left(N_{X, Y}\right)$. Moreover, each fiber of $N_{X, Y}$ is a complex $T$-module with non-zero weights. Denoting by $\operatorname{det}\left(N_{Y, X}\right)$ the product of these weights, we have in $H_{T}^{*}(Y)=S \otimes H^{*}(Y)$ :

$$
c_{n, T}\left(N_{Y, X}\right) \equiv \operatorname{det}\left(N_{Y, X}\right) \otimes[Y] \quad\left(\bmod S \otimes H^{+}(Y)\right) .
$$

Because $S \otimes H^{+}(Y)$ consists of nilpotent elements, $c_{n, T}\left(N_{Y, X}\right)$ becomes invertible in $H_{T}^{*}(Y)$ after inverting $\operatorname{det}\left(N_{Y, X}\right)$. Moreover, setting $i_{T *}:=\sum_{Y} i_{Y *}$, we have

$$
i_{T}^{*}\left(i_{T *}(u)\right)=\sum_{Y} c_{t o p, T}\left(N_{Y, X}\right) \cup u
$$

for any $u \in H_{T}^{*}\left(X^{T}\right)$. As a consequence, $i_{T *}$ is an isomorphism after localization, with inverse

$$
u \mapsto \sum_{Y} c_{t o p, T}\left(N_{Y, X}\right)^{-1} \cup i_{Y}^{*}(u) .
$$

This "covariant" version of the localization theorem will be extended in Section 4 to equivariant Chow groups, which can be considered as algebraic equivariant homology groups. It has many important applications to residue or integral formulae, in particular the Bott residue formula and the Duistermaat-Heckman theorem, for which we refer to [1] and [5]. 


\section{A precise version of the localization theo- rem}

As before, we consider a compact torus $T$ and a $T$-space $X$; we denote by $i_{T}: X^{T} \rightarrow X$ the inclusion of the fixed point set. We wish to describe the image of the restriction map

$$
i_{T}^{*}: H_{T}^{*}(X) \rightarrow H_{T}^{*}\left(X^{T}\right)
$$

For this, let $T^{\prime} \subset T$ be a subtorus; observe that

$$
i_{T}: X^{T} \rightarrow X
$$

factors as

$$
i_{T, T^{\prime}}: X^{T} \rightarrow X^{T^{\prime}}
$$

followed by

$$
i_{T^{\prime}}: X^{T^{\prime}} \rightarrow X
$$

Thus, the image of $i_{T}^{*}$ is contained in the image of $i_{T, T^{\prime}}^{*}$. This observation will lead to a complete description of the image of $i_{T}^{*}$, if the $S$-module $H_{T}^{*}(X)$ is free. Indeed, we have the following result, due to Chang-Skjelbred 29 and Hsiang [31] Corollary p. 63, in a slightly different formulation.

Theorem 6 Let $X$ be a T-space which admits an equivariant embedding into the space of a finite-dimensional representation of $T$. If the $S$-module $H_{T}^{*}(X)$ is free, then the map

$$
i_{T}^{*}: H_{T}^{*}(X) \rightarrow H_{T}^{*}\left(X^{T}\right)
$$

is injective, and its image is the intersection of the images of the maps

$$
i_{T, T^{\prime}}^{*}: H_{T}^{*}\left(X^{T^{\prime}}\right) \rightarrow H_{T}^{*}\left(X^{T}\right)
$$

where $T^{\prime}$ runs over all subtori of codimension 1 of $T$.

Proof We already know that $i_{T}^{*}$ becomes an isomorphism after inverting a finite family $\mathcal{F}$ of non-trivial characters of $T$. Because the $S$-module $H_{T}^{*}(X)$ is free, it follows that $i_{T}^{*}$ is injective.

It remains to prove that the intersection of the images of the $i_{T, T^{\prime}}^{*}$ is contained in the image of $i_{T}^{*}$. Choose a basis $\left(e_{j}\right)_{j \in J}$ of the free $S$-module $H_{T}^{*}(X)$. For any $j \in J$, let

$$
e_{j}^{*}: H_{T}^{*}(X) \rightarrow S
$$


be the corresponding coordinate function. Then there exists a $S$-linear map

$$
f_{j}: H_{T}^{*}\left(X^{T}\right) \rightarrow S[1 / \chi]_{\chi \in \mathcal{F}}
$$

such that $f_{j} \circ i_{T}^{*}=e_{j}^{*}$.

We may assume that each $\chi \in \mathcal{F}$ is primitive, i.e., not divisible in $\Xi(T)$. Then its kernel $\operatorname{ker}(\chi) \subset T$ is a subtorus of codimension 1 . Let $u$ be in the image of $i_{T, k e r(\chi)}^{*}$; write

$$
u=i_{T, k e r(\chi)}^{*}(v)
$$

where $v \in H_{T}^{*}\left(X^{k e r}(\chi)\right)$. By Lemma $⿴$ applied to $\Gamma=\operatorname{ker}(\chi)$, there exist a product $P_{\chi}$ of weights of $T$ which are not multiples of $\chi$, such that $P_{\chi} v$ is in the image of $i_{k e r(\chi)}^{*}$. It follows that $P_{\chi} u$ is in the image of $i_{T}^{*}$. Applying $f_{j}$, we obtain $P_{\chi} f_{j}(u) \in S$. Thus, the denominator of $f_{j}(u)$ is not divisible by $\chi$.

If $u \in H_{T}^{*}\left(X^{T}\right)$ is in the intersection of the images of the $i_{T, k e r(\chi)}^{*}$ for all $\chi \in \mathcal{F}$, then $f_{j}(u) \in S[1 / \chi]_{\chi \in \mathcal{F}}$ but the denominator of $f_{j}(u)$ is not divisible by any element of $\mathcal{F}$; whence $f_{j}(u) \in S$. It follows that $u=i_{T}^{*}\left(\sum_{j \in J} f_{j}(u) e_{j}\right)$ is in the image of $i_{T}^{*}$.

Observe that the assumptions of Theorem 6 are satisfied whenever $X$ is a compact Hamiltonian $T$-space. Indeed, the $S$-module $H_{T}^{*}(X)$ is free by Proposition 2, and the fixed point set of any closed subgroup of $T$ is a Hamiltonian $T$-space by Lemma 5 .

If moreover $X^{T}$ is finite and each $X^{T^{\prime}}$ has dimension at most 2, then each connected component $Y$ of $X^{T^{\prime}}$ is either a point or a 2-sphere, see e.g. [5] Chapter I, 3.3. In the latter case, we can see $Y$ as complex projective line where $T$ acts through multiplication by a character $\chi$; then $Y^{T}=\{0, \infty\}$. It is easily checked that

$$
i_{T}^{*} H_{T}^{*}(Y)=\left\{\left(f_{0}, f_{\infty}\right) \in S \times S \mid f_{0} \equiv f_{\infty}(\bmod \chi)\right\} .
$$

From the discussion above, we deduce the following explicit description of the image of $H_{T}^{*}(X)$ under restriction to the fixed point set, for certain Hamiltonian $T$-spaces $X$ [27].

Corollary 7 Let $X$ be a compact Hamiltonian T-space with finitely many fixed points $x_{1}, \ldots, x_{m}$ and such that $\operatorname{dim}\left(X^{T^{\prime}}\right) \leq 2$ for any subtorus $T^{\prime} \subset T$ of codimension 1. Then, via $i_{T}^{*}$, the algebra $H_{T}^{*}(X)$ is isomorphic to the subalgebra of $S^{m}$ consisting of all $m$-tuples $\left(f_{1}, \ldots, f_{m}\right)$ such that:

$$
f_{j} \equiv f_{k} \quad(\bmod \chi)
$$


whenever the fixed points $x_{j}$ and $x_{k}$ are in the same connected component of $X^{k e r}(\chi)$, for $\chi$ a primitive character of $T$. Moreover, the cohomology algebra $H^{*}(X)$ is the quotient of $H_{T}^{*}(X)$ by its ideal generated by all $(f, f, \ldots, f)$ where $f \in S$ is homogeneous of positive degree.

This statement can be reformulated in a more geometric way, by introducing the affine algebraic scheme $V(X)$ over $\mathbb{Q}$ associated with the finitely generated $\mathbb{Q}$-algebra $H_{T}^{*}(X)$. Indeed, $V(X)$ is obtained from the disjoint union of $m$ copies of $\mathfrak{t}$ by identifying the $j$-th and $k$-th copies along their common hyperplane $(\chi=0)$ whenever $x_{j}$ and $x_{k}$ are in the same connected component of $X^{k e r(\chi)}$. In particular, $V(X)$ is reduced. The inclusion $S \subset H_{T}^{*}(X)$ defines a morphism $V(X) \rightarrow \mathfrak{t}$ which restricts to the identity on each copy of $\mathfrak{t}$. This morphism is finite and flat (because $H_{T}^{*}(X)$ is a finite free $S$-module) and its scheme-theoretic fiber at the origin is the scheme associated with $H^{*}(X)$.

\section{Examples}

1) (flag manifolds) Let $G$ be a compact connected Lie group, $T \subset G$ a maximal torus, and $X=G / T$ the flag manifold of the complexification of $G$. Denote by $x$ the base point of $X$. Then the fixed point set $X^{T}$ is the orbit $W x$; it identifies to the Weyl group $W$. Denote by $\Phi$ the root system of $(G, T)$. Let $\chi$ be a primitive character of $T$. If $\chi$ is not in $\Phi$, then $X^{\operatorname{ker}(\chi)}=X^{T}$. If $\chi$ is in $\Phi$, with corresponding reflection $s \in W$, then

$$
X^{\operatorname{ker}(\chi)}=G^{k e r(\chi)} W x
$$

is a disjoint copy of $|W| / 2$ complex projective lines joigning the fixed points $w x$ and $s w x$ for all $w \in W$. So we obtain from the corollary:

$$
H_{T}^{*}(G / T)=\left\{\left(f_{w}\right)_{w \in W} \mid f_{w} \in S, f_{w} \equiv f_{s_{\alpha} w}(\bmod \alpha) \forall \alpha \in \Phi, \forall w \in W\right\} .
$$

In other words, the scheme $V(G / T)$ is the union of $|W|$ copies of $\mathfrak{t}$ identified along all reflection hyperplanes.

On the other hand, we saw that $H_{T}^{*}(G / T)=S \otimes_{S^{W}} S$. This can be related to the description above, as follows. The homomorphism $S \otimes 1 \rightarrow H_{T}^{*}(G / T)$ is given by the structure of $S$-module of $H_{T}^{*}(G / T)$; its composition with $i_{T}^{*}$ maps each $f \in S$ to the tuple $(f, f, \ldots, f)$. The homomorphism $1 \otimes S \rightarrow H_{T}^{*}(G / T)$ is the characteristic homomorphism; its composition with $i_{T}^{*}$ maps $f$ to the tuple $(w(f))_{w \in W}$. The scheme $V(G / T)$ identifies to the union of subspaces 
of $\mathfrak{t} \times \mathfrak{t}$ which are images of the diagonal $\Delta(\mathfrak{t})$ by some element of $W \times W$. Indeed, we have

$$
V(X)=\operatorname{Spec}\left(S \otimes_{S^{W}} S\right)=\mathfrak{t} \times_{\mathfrak{t} / W} \mathfrak{t}=\{(a, b) \in \mathfrak{t} \times \mathfrak{t} \mid a \in W b\} .
$$

This is also the union of $|W|$ copies of $\mathfrak{t}$ identified along reflection hyperplanes.

For other pictures of the equivariant cohomology ring of the flag manifold, we refer to [2], [3] and [39]; see also Section 4 and Peterson's lecture notes in this volume.

2) (toric manifolds) Let $X$ be a projective toric manifold, that is, $X$ is a complex algebraic projective manifold and the complexification $T^{\mathbb{C}}$ of $T$ acts on $X$ with a dense orbit isomorphic to $T^{\mathbb{C}}$. Then $X$ is a Hamiltonian $T$-space, $\left.\mu\right|_{X^{T}}$ is injective, and $\mu(X):=P$ is a convex polytope in $\mathfrak{t}^{*}$ with vertex set $\mu\left(X^{T}\right):=\mathcal{V}$. Moreover, $\mu$ induces a bijection bewteen $T^{\mathbb{C}}$-orbit closures in $X$ and faces of $P$ [5] Chapter 4 . It follows that, for a character $\chi$ of $T$, the set $X^{\operatorname{ker}(\chi)}$ is equal to $X^{T}$, except when there exists an edge of $P$ with direction $\chi$. In this case, $X^{\operatorname{ker}(\chi)}$ is a complex projective line joigning the $T$-fixed points associated with the vertices of this edge. Thus, $i_{T}^{*} H_{T}^{*}(X)$ consists of all families $\left(f_{v}\right)_{v \in \mathcal{V}}$ of $S$, such that $f_{v} \equiv f_{v^{\prime}}(\bmod \chi)$ whenever the segment $\left[v, v^{\prime}\right]$ is an edge of $P$ with direction $\chi$.

Let $F$ be a face of $P$. Choose a point $x_{F}$ in the relative interior of $F$, then the union of all half-lines with origin at $x_{F}$ and which meet $P \backslash x_{F}$ is a closed convex cone which depends only on $F$; we denote this cone by $C_{F} P$. The translated cone $-x_{F}+C_{F} P$ only depends on $F$, too, and contains the origin of $\mathfrak{t}^{*}$; denote by $\sigma_{F} \subset \mathfrak{t}$ its dual cone. Then the cones $\sigma_{F}$ are a subdivision of $\mathfrak{t}$, with maximal cones $\sigma_{v}(v \in \mathcal{V})$.
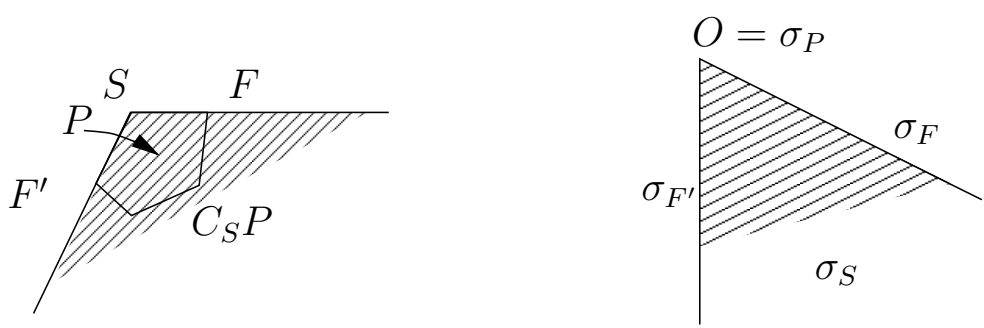

We can consider each $f_{v} \in S$ as a polynomial function on $\sigma_{v}$. Then the congruences above mean that the functions $f_{v}$ are compatible along all common facets of the cones $\sigma_{v}$, that is, they glue together into a continuous function on $\mathfrak{t}$. We conclude that $H_{T}^{*}(X)$ is identified with the algebra of 
continuous functions on $\mathfrak{t}$ which are piecewise polynomial with respect to the subdvision $\left(\sigma_{F}\right)$ ( $F$ a face of $P$ ). Moreover, the image of $S$ in $H_{T}^{*}(X)$ is identified to the algebra of polynomial functions. We refer to [13 for more on the relations between continuous, piecewise polynomial functions and toric manifolds.

We now generalize this description to the class of all compact multiplicityfree Hamiltonian spaces, in the following sense.

Definition [29], [47] A Hamiltonian $G$-space $X$ is multiplicity-free if $X$ is connected and the preimage under the moment map of any coadjoint $G$-orbit consists of finitely many $G$-orbits.

If moreover $X$ is compact, then the fibers of the moment map are connected (see 42 for a simple proof of this fact). Thus, multiplicity-free amounts to: the preimage of each orbit under the moment map is an unique orbit.

By [12], a complex projective $G$-manifold $X$ is multiplicity-free if and only if it is spherical for the action of the complexification $G^{\mathbb{C}}$, that is, a Borel subgroup of $G^{\mathbb{C}}$ has a dense orbit in $X$.

For compact multiplicity-free spaces, we have the following sharper version of Lemma 5 .

Lemma 8 Let $X$ be a compact multiplicity-free $G$-space and $\Gamma \subset G$ a closed subgroup. Then $X^{\Gamma}$ has only finitely many components, and each of them is a compact multiplicity-free $G^{\Gamma}$-space. In particular, the fixed point set of a maximal torus of $G$ is finite. Moreover, denoting by $X_{1}$ the set of all $x \in X$ such that the rank of the isotropy group $G_{x}$ is at least $r k(G)-1$, and identifying $\left(\mathfrak{g}^{*}\right)^{T}$ with $\mathfrak{t}^{*}$, the intersection $\mu\left(X_{1}\right) \cap \mathfrak{t}^{*}$ is a finite union of segments with ends in $\mu\left(X^{T}\right)$.

Proof Let $Y$ be a component of $X^{\Gamma}$ and let $x \in Y$. Then we know that $\mu^{-1}(\mu(G x))=G x$. Thus, $\left.\mu\right|_{Y} ^{-1}\left(\mu\left(G^{\Gamma} x\right)\right)$ is contained in $(G x)^{\Gamma}$. But the set $(G x)^{\Gamma}$ is a finite union of orbits of $G^{\Gamma}$. It follows that $Y$ is multiplicity-free.

In particular, each component $Y$ of $X^{T}$ is a multiplicity-free space for the action of $G^{T}=T$. Thus, $Y$ must be a point.

For the last assertion, observe first that $X_{1}$ is the union of the sets $G X^{T^{\prime}}$ where $T^{\prime} \subset T$ is a subtorus of codimension 1. Moreover, the number of subsets $X^{T^{\prime}}$ is finite (this can be seen by linearizing the action of $T$ around 
fixed points). Thus, it is enough to check that each $\mu\left(G X^{T^{\prime}}\right) \cap \mathfrak{t}^{*}$ is a finite union of segments with ends in $\mu\left(X^{T}\right)$. But

$$
\mu\left(G X^{T^{\prime}}\right) \cap \mathfrak{t}^{*}=G \mu\left(X^{T^{\prime}}\right) \cap \mathfrak{t}^{*}
$$

and $\mu\left(X^{T^{\prime}}\right)$ is contained in $\left(\mathfrak{g}^{*}\right)^{T^{\prime}}=G^{T^{\prime}} \mathfrak{t}^{*}$, whence

$$
\mu\left(G X^{T^{\prime}}\right) \cap \mathfrak{t}^{*}=W\left(\mu\left(X^{T^{\prime}}\right) \cap \mathfrak{t}^{*}\right) .
$$

So we can replace $(G, X)$ by $\left(G^{T^{\prime}} / T^{\prime}, Y\right)$ where $Y$ is a component of $X^{T^{\prime}}$, and thus we can assume that the rank of $G$ is one. Then $\mu(X) \cap \mathfrak{t}^{*}$ is a segment, or a union of two segments with ends in $\mu\left(X^{T}\right)$ [33].

Observe that $W$ acts on $X^{T}$ and that both $\mu\left(X^{T}\right)$ and $\mu\left(X_{1}\right) \cap \mathfrak{t}^{*}$ are $W$-invariant subsets of $\mathfrak{t}^{*}$. Choose a Weyl chamber $\mathfrak{t}_{+}^{*} \subset \mathfrak{t}^{*}$ and set

$$
\mu\left(X^{T}\right) \cap \mathfrak{t}_{+}^{*}=\left\{\lambda_{1}, \ldots, \lambda_{m}\right\} .
$$

Then $\mu\left(X^{T}\right)=W \lambda_{1} \cup \cdots \cup W \lambda_{m}$. For $1 \leq j \leq m$, choose $x_{j} \in X^{T}$ such that $\mu\left(x_{j}\right)=\lambda_{j}$ and denote by $W_{j}$ the isotropy group of $x_{j}$ in $W$. Then the $W$-orbits in $X^{T}$ are the cosets $W / W_{j}(1 \leq j \leq m)$. As a consequence, we have an isomorphism (by Proposition 1)

$$
H_{T}^{*}\left(X^{T}\right)^{W} \simeq \prod_{j=1}^{m} S^{W_{j}} .
$$

We now describe the image of $H_{G}^{*}(X) \simeq H_{T}^{*}(X)^{W}$ in $H_{T}^{*}\left(X^{T}\right)^{W}$ under restriction to fixed points $i_{T}^{*}$.

Theorem 9 Let $X$ be a compact multiplicity-free space under a compact connected Lie group $G$. Then, with notation as above, the algebra $H_{G}^{*}(X)$ is isomorphic via $i_{T}^{*}$ to the subalgebra of $S^{m}$ consisting of all $m$-uples $\left(f_{1}, \ldots, f_{m}\right)$ such that:

1) each $f_{j}$ is in $S^{W_{j}}$, and

2) $f_{j} \equiv w\left(f_{k}\right)\left(\bmod \lambda_{j}-w\left(\lambda_{k}\right)\right)$ whenever $w \in W$ and the segment $\left[\lambda_{j}, w\left(\lambda_{k}\right)\right]$ is a component of $\mu\left(X_{1}\right) \cap \mathfrak{t}^{*}$.

Moreover, $i_{T}^{*}\left(S^{W}\right)$ consists of all tuples $(f, \ldots, f)$ where $f \in S^{W}$.

Proof Let $\Gamma \subset T$ be a subtorus of codimension one. Let $\chi$ be a character of $T$ with kernel $\Gamma$ and let $Y$ be a connected component of $X^{\Gamma}$. By Lemma 8 , 
the $G^{\Gamma}$-variety $Y$ is multiplicity-free. Set $G^{\Gamma} / \Gamma:=H$; then $H$ is a compact connected Lie group of rank 1, and thus it is isomorphic to $S^{1}, \mathrm{SU}(2)$ or $\mathrm{SO}(3)$. Therefore, the $H$-multiplicity free space $Y$ must be of dimension at most 4. Two cases can occur:

1) $Y$ is two-dimensional. Then $Y$ is isomorphic to complex projective line, and $Y^{T}$ consists of two fixed points $y, z$. Restriction to these fixed points identifies $H_{T}^{*}(Y)$ to the set of all $\left(f_{y}, f_{z}\right) \in S \times S$ such that

$$
f_{y} \equiv f_{z}(\bmod \chi)
$$

If $\chi$ is not a root of $(G, T)$, then $H \simeq S^{1}$ and $\mu(Y)$ is the segment $[\mu(y), \mu(z)]$ in $\mathfrak{t}^{*}$. Thus, this segment lies in $\mu\left(X_{1}\right) \cap \mathfrak{t}^{*}$. On the other hand, if $\chi$ is a root, let $s \in W$ be the corresponding reflection and let $\tilde{s}$ be a representative of $s$ in the normalizer of $T$. Then $Y$ is invariant under $\tilde{s}$, and

$$
H_{T}^{*}(Y)^{s}=\left\{\left(f_{y}, s\left(f_{y}\right)\right) \mid f_{y} \in S\right\}
$$

(indeed, $f-s(f)$ is divisible by $\chi$ for any $f \in S$ ).

2) $Y$ is four-dimensional. Then $H$ is isomorphic to $\mathrm{SU}(2)$ or $\mathrm{SO}(3)$, and the $H$-variety $Y$ is either a rational ruled surface, or the projectivization of a three-dimensional complex representation of $\mathrm{SU}(2)$ (see [33] and [5] Chapter IV, Appendix A). In the former case, $Y^{T}$ consists of four points $y, s(y), z$, $s(z)$ where $s$ is the non-trivial element of the Weyl group of $(H, T / \Gamma)$; we may assume that the segment $[\mu(y), \mu(z)]$ lies in $\mu(Y) \cap \mathfrak{t}^{*}$. It is easy to check that restriction to fixed points maps $H_{T}^{*}(Y)$ onto the set of all quadruples $\left(f_{y}, f_{s(y)}, f_{z}, f_{s(z)}\right) \in S^{4}$ such that

$$
f_{y} \equiv f_{s(y)} \equiv f_{z} \equiv f_{s(z)}(\bmod \chi), f_{y}+f_{s(y)} \equiv f_{z}+f_{s(z)}\left(\bmod \chi^{2}\right) .
$$

It follows that

$$
H_{T}^{*}(Y)^{s}=\left\{\left(f_{y}, f_{z}\right) \in S \times S \mid f_{y} \equiv f_{z}(\bmod \chi)\right\} .
$$

In the latter case, we have similarly $Y^{T}=\{y, s(y), z\}$ where $z=s(z)$, and $H_{T}^{*}(Y)$ consists of the triples $\left(f_{y}, f_{s(y)}, f_{z}\right) \in S^{3}$ such that

$$
f_{y} \equiv f_{s(y)} \equiv f_{z}(\bmod \chi), f_{y}+f_{s(y)} \equiv 2 f_{z}\left(\bmod \chi^{2}\right) .
$$

It follows that

$$
H_{T}^{*}(Y)^{s}=\left\{\left(f_{y}, f_{z}\right) \in S \times S^{s} \mid f_{y} \equiv f_{z}(\bmod \chi)\right\}
$$


We conclude that the image of $i_{T}^{*}$ is defined by our congruences. Observe that the image of $i_{T}^{*}: H_{T}^{*}(X) \rightarrow H_{T}^{*}\left(X^{T}\right)$ is defined by congruences of the form (1), (2) or (3).

\section{Examples}

1) (coadjoint orbits) Let $X$ be the $G$-orbit of $\lambda \in \mathfrak{g}^{*}$; we may assume that $\lambda \in \mathfrak{t}_{+}^{*}$. Then $\mu: X \rightarrow \mathfrak{g}^{*}$ is the inclusion map, whence $\mu\left(X^{T}\right) \cap \mathfrak{t}^{*}=\{\lambda\}$ and $\mu\left(X_{1}\right) \cap \mathfrak{t}^{*}=W \cdot \lambda$. Then Theorem 9 reduces to the isomorphism

$$
H_{G}^{*}(G \lambda)=S^{W_{\lambda}}
$$

which follows more directly from the isomorphism

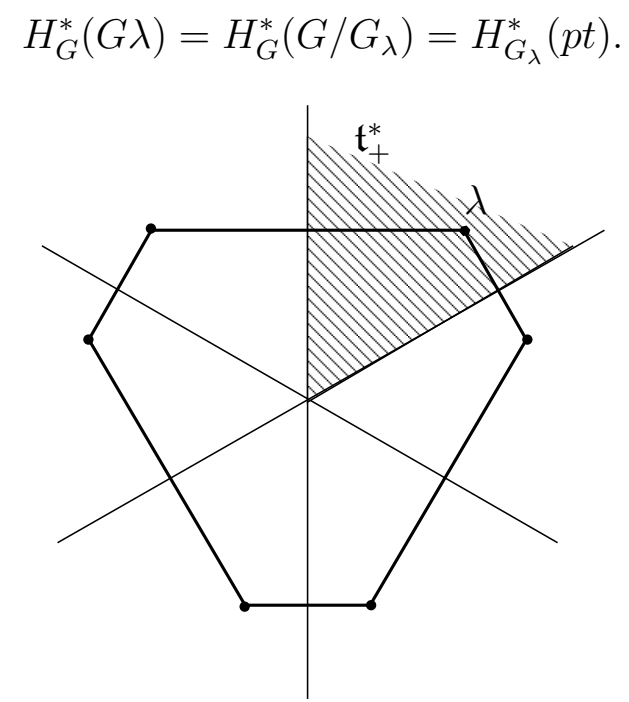

2) (toric varieties). If $T$ is a torus and $X$ a projective toric manifold for the complexification $T^{\mathbb{C}}$, then the set $\mu\left(X_{1}\right) \cap \mathfrak{t}^{*}$ is the union of all edges of the polytope $\mu(X)$. So Theorem 9 gives back the description of $H_{T}^{*}(X)$ found in the second example of this section.

3) (complete conics). Let $V$ be the vector space of quadratic forms on $\mathbb{C}^{3}$, let $V^{*}$ be the dual space, and let $\mathbb{P}=\mathbb{P}(V) \times \mathbb{P}\left(V^{*}\right)$ be the product of their projectivizations. Let $X \subset \mathbb{P}$ be the closure of the set of classes $\left([A],\left[A^{-1}\right]\right)$ where $A \in V$ is non-degenerate and $A^{-1} \in V^{*}$ is the dual quadratic form. Then $X$ is a complex projective manifold, called the space of complete conics. Moreover, $X$ is spherical for the natural action of $\operatorname{GL}(3)$, and hence multiplicity-free for the action of the maximal compact subgroup $G:=U(3)$. 
The set $\mu\left(X_{1}\right) \cap \mathfrak{t}^{*}$ is given by the following picture.

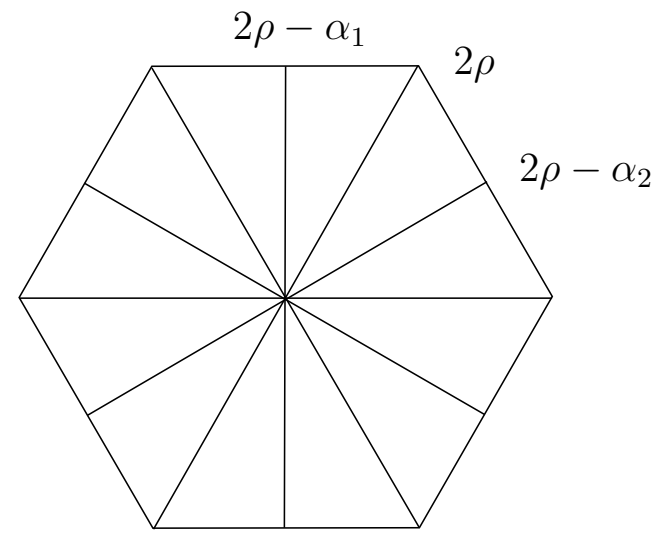

It follows that the algebra $H_{G}^{*}(X)$ consists of all triples $\left(f, f_{1}, f_{2}\right)$ in $S \times S^{s_{1}} \times S^{s_{2}}$ such that $f \equiv f_{1}\left(\bmod \alpha_{1}\right), f \equiv f_{2}\left(\bmod \alpha_{2}\right)$ and that $f_{1} \equiv s_{\alpha_{1}+\alpha_{2}}\left(f_{2}\right)\left(\bmod 2 \alpha_{1}+\alpha_{2}\right)$ where $\alpha_{1}, \alpha_{2}$ are the simple roots, with corresponding reflections $s_{1}, s_{2}$.

The variety of complete conics is the basic example of a "complete symmetric variety", a nice compactification of a symmetric space [18]. The cohomology ring of complete symmetric varieties is described by other methods in [7] and [43].

\section{Equivariant Chow groups}

We begin by recalling the definition and some basic features of (usual) Chow groups; a complete exposition can be found in [24].

Let $X$ be a scheme of finite type over the field of complex numbers (most of what follows holds more generally over any algebraically closed field). The group of algebraic cycles on $X$ is the abelian group $Z_{*}(X)$ freely generated by symbols $[Y]$ where $Y \subset X$ is a subvariety (that is, a closed subscheme which is a variety). Observe that the group $Z_{*}(X)$ is graded by dimension.

For $Y$ as before, and for a rational function $f$ on $Y$, we define the divisor of $f$, an element of $Z_{*}(X)$, by

$$
\operatorname{div}(f):=\sum_{D} \operatorname{ord}_{D}(f)[D]
$$

(sum over all prime divisors $D \subset Y$ ), where $\operatorname{ord}_{D}(f)$ denotes the order of the 
zero or pole of $f$ along $D$. The cycles $\operatorname{div}(f)$ for $Y$ and $f$ as before, generate a graded subgroup $R_{a t}(X) \subset Z_{*}(X)$ : the group of rationally trivial cycles.

By definition, the Chow group $A_{*}(X)$ is the quotient $Z_{*}(X) / \operatorname{Rat}_{*}(X)$; it is a graded abelian group with homogeneous components $A_{n}(X)$ where $0 \leq n \leq \operatorname{dim}(X)$. The top degree component $A_{\operatorname{dim}(X)}(X)$ is freely generated by the top dimensional irreducible components of $X$.

If $X$ is equidimensional (that is, if all irreducible components of $X$ have the same dimension), then we set $A^{n}(X):=A_{\operatorname{dim}(X){ }_{n}}(X)$, the group of cycles of codimension $n$, and we denote by $A^{*}(X)$ the Chow group graded by codimension. If moreover $X$ is smooth, then there is an intersection product on $A^{*}(X)$ which makes it a graded ring.

We now review the functorial properties of Chow groups. Any proper morphism $\pi: X \rightarrow Y$ defines a push-forward homomorphism $\pi_{*}: A_{*}(X) \rightarrow$ $A_{*}(Y)$ which preserves degree. On the other hand, a flat morphism $\pi$ : $X \rightarrow Y$ with $d$-dimensional fibers induces a pull-back homomorphism $\pi^{*}$ : $A_{*}(Y) \rightarrow A_{*}(X)$ which increases degree by $d$. If moreover all fibers are isomorphic to affine space, then $\pi^{*}$ is an isomorphism [26]. In particular, the projection of a vector bundle induces isomorphisms on Chow groups. This can be seen as a version of homotopy invariance. Finally, any local complete intersection morphism $\pi: X \rightarrow Y$ of codimension $d$ (in the sense of 24 6.6) induces a homomorphism $\pi^{*}: A_{*}(Y) \rightarrow A_{*}(X)$ which decreases degree by $d$. In particular, if $X$ and $Y$ are smooth, then any morphism $\pi: X \rightarrow Y$ defines a ring homomorphism $\pi^{*}: A^{*}(Y) \rightarrow A^{*}(X)$ which preserves degree.

For a scheme $X$ and a closed subscheme $Y$, we have a short exact sequence

$$
A_{*}(Y) \rightarrow A_{*}(X) \rightarrow A_{*}(X \backslash Y) \rightarrow 0 .
$$

It can be extended to a long exact sequence by introducing higher Chow groups (which will not be considered here).

Any vector bundle $E$ on $X$ has Chern classes $c_{j}(E)$, which are homogeneous operators on $A_{*}(X)$ of respective degree $-j$. If $X$ is smooth and $i: Y \rightarrow X$ denotes the inclusion of a smooth subvariety of codimension $d$, then the composition $i^{*} \circ i_{*}$ is multiplication by $c_{d}\left(N_{X, Y}\right)$, the top Chern class of the normal bundle to $Y$ in $X$ (this holds more generally if $Y$ is a local complete intersection in $X$ ).

Each subvariety $Y \subset X$ has a homology class $c l_{X}(Y)$ of degree $2 \operatorname{dim}(Y)$ in Borel-Moore homology $H_{*}(X, \mathbb{Z})$ [24] 19.1. The assignement $Y \mapsto c l_{X}(Y)$ defines a cycle map

$$
c l_{X}: A_{*}(X) \rightarrow H_{*}(X, \mathbb{Z})
$$


which doubles degrees. If moreover $X$ is smooth of (complex) dimension $N$, then $H_{j}(X, \mathbb{Z})=H^{2 N-j}(X, \mathbb{Z})$ and thus, we have a cycle map

$$
c l_{X}: A^{*}(X) \rightarrow H^{*}(X, \mathbb{Z}),
$$

which is a (degree doubling) ring homomorphism. The cycle map is an isomorphism if $X$ has a cellular decomposition [24] Example 19.1.11. But in general, the cycle map is far from being injective or surjective.

\section{Examples}

1) (curves) Let $X$ be a smooth projective curve of genus $g$. Then $A_{1}(X) \simeq$ $\mathbb{Z}$ is freely generated by $[X]$, and is isomorphic to $H_{2}(X, \mathbb{Z})$. The cycle map $A_{0}(X) \rightarrow H_{0}(X, \mathbb{Z})$ is the degree map; its kernel is the Jacobian variety of $X$, a compact torus of dimension $2 g$. The cokernel of the cycle map $A_{*}(X) \rightarrow H_{*}(X, \mathbb{Z})$ is the group $H_{1}(X, \mathbb{Z})$, isomorphic to $\mathbb{Z}^{2 g}$.

2) (linear algebraic groups) Let $G$ be a connected linear algebraic group and $B \subset G$ be a Borel subgroup. Then the flag manifold $G / B$ has a cellular decomposition by Schubert cells, and thus its Chow ring is isomorphic to its integral cohomology ring. Let $T \subset B$ be a maximal torus and let $\Xi(T)$ be its character group. Then we have the characteristic homomorphism $c: \Xi(T) \rightarrow$ $A^{1}(G / B)$ which maps each character to the Chern class of the associated line bundle over $G / B$. It extends to an algebra homomorphism

$$
c: S_{\mathbb{Z}} \rightarrow A^{*}(G / B)
$$

where $S_{\mathbb{Z}}$ is the symmetric algebra of $\Xi(T)$ over the integers.

We can choose a maximal compact subgroup $G_{c} \subset G$ such that the group $T \cap G_{c}=: T_{c}$ is a maximal compact torus of $T$; then the map $G_{c} / T_{c} \rightarrow G / B$ is an homeomorphism. Now, by Proposition 1, the characteristic homomorphism induces an isomorphism

$$
S /\left(S_{+}^{W}\right) \rightarrow A^{*}(G / B)_{\mathbb{Q}}
$$

Moreover, the Chow ring of $G$ is isomorphic to the quotient of $A^{*}(G / B)$ by its ideal generated by $c(\Xi(T))$ 28] p. 21. To see this, choose a basis $\left(\chi_{1}, \ldots, \chi_{\ell}\right)$ of $\Xi(T)$. Consider the action of $T$ on $\mathbb{C}^{\ell}$ with weights $\chi_{1}, \ldots, \chi_{\ell}$. Then $T$ embeds into $\mathbb{C}^{\ell}$ as the complement of the coordinate hyperplanes. Let $E:=G \times_{T} \mathbb{C}^{\ell}$ be the associated vector bundle over $G / T$. Then $E$ is a direct sum of line bundles $L_{1}, \ldots, L_{\ell}$, and $G=G \times_{T} T$ embeds into $E$ as the 
complement of the union of zero sections $E^{(j)}:=\oplus_{i \neq j} L_{i}(1 \leq j \leq \ell)$. Using the exact sequence

$$
\oplus_{j=1}^{\ell} A^{*}\left(E^{(j)}\right) \rightarrow A^{*}(E) \rightarrow A^{*}(G)
$$

and the fact that the image of $A^{*}\left(E^{(j)}\right)$ in $A^{*}(E)$ is the ideal generated by $\left[E^{(j)}\right]$, we see that

$$
\begin{array}{r}
A^{*}(G)=A^{*}(E) /\left(\left[E^{(1)}\right], \ldots,\left[E^{(\ell)}\right]\right)=A^{*}(G / T) /\left(c\left(\chi_{1}\right), \ldots, c\left(\chi_{\ell}\right)\right) \\
=A^{*}(G / B) /(c(\Xi(T))) .
\end{array}
$$

In particular, because $c$ is surjective over $\mathbb{Q}$, the positive degree part of $A^{*}(G)$ is finite.

On the other hand, the rational cohomology algebra $H^{*}(G)$ is a free exterior algebra on $\ell$ generators [30]. Thus, the cycle map $c l_{G}$ is not an isomorphism over $\mathbb{Q}$, except when $G$ is unipotent.

Now we introduce the equivariant Chow groups, after Edidin and Graham 222. Let $G$ be a linear algebraic group and let $n$ be a non negative integer. As in Example 3 of the first section, we can find a $G$-module $V$ and a $G$-invariant open subset $U \subset V$ (depending on $n$ ) satisfying the following conditions.

1) The quotient $U \rightarrow U / G$ exists and is a principal $G$-bundle.

2) The codimension of $V \backslash U$ in $V$ is larger than $n$.

Observe that $U \rightarrow U / G$ is an approximation of the universal $G$-bundle by an algebraic bundle.

Now let $X$ be a scheme with a $G$-action such that $X$ can be covered by invariant quasi-projective open subsets (by [46], this assumption is fulfilled when $X$ is normal); we will say that $X$ is a $G$-scheme. Then the quotient of $X \times U$ by the diagonal action of $G$ exists as a scheme; we denote this quotient by $X \times{ }_{G} U$.

If $X$ is equidimensional, we define its equivariant Chow group of degree $n$ by

$$
A_{G}^{n}(X):=A^{n}\left(X \times_{G} U\right) .
$$

This makes sense because $X \times{ }_{G} U$ is equidimensional, and also because this does not depend on the choice of $U$. Indeed, if $U^{\prime} \subset V^{\prime}$ is another choice, then the quotient of $X \times U \times V^{\prime}$ by the diagonal action of $G$ exists and defines a map

$$
p:\left(X \times U \times V^{\prime}\right) / G \rightarrow X \times_{G} U \text {. }
$$


Observe that $p$ is smooth with fibers isomorphic to $V^{\prime}$. Thus, we have isomorphisms

$$
A^{n}\left(X \times_{G} U\right) \simeq A^{n}\left(\left(X \times U \times V^{\prime}\right) / G\right) \simeq A^{n}\left(\left(X \times U \times U^{\prime}\right) / G\right),
$$

the latter being a consequence of assumption (2) for $U^{\prime}$. It follows that $A^{n}\left(X \times_{G} U\right) \simeq A^{n}\left(X \times{ }_{G} U^{\prime}\right)$.

For $X$ not necessarily equidimensional, we set

$$
A_{n}^{G}(X):=A_{n-\operatorname{dim}(G)+\operatorname{dim}(U)}\left(X \times_{G} U\right) .
$$

Arguing as above, we see that this group is independent of $U$, so that we can define the equivariant Chow group

$$
A_{*}^{G}(X)=\bigoplus_{n \in \mathbb{Z}} A_{n}^{G}(X) .
$$

By definition, we have $A_{n}^{G}(X)=0$ for $n>\operatorname{dim}(X)$; but $A_{n}^{G}(X)$ may be non trivial for $n<0$. For equidimensional $X$, we have

$$
A_{G}^{n}(X)=A_{\operatorname{dim}(X)-n}^{G}(X) .
$$

We now list some properties of equivariant Chow groups; let us mention first that they satisfy the functorial properties of usual Chow groups, and that each linearized vector bundle has equivariant Chern classes.

If $X$ is smooth, then the same holds for each $X \times_{G} U$. It follows that $A_{G}^{*}(X)$ is a graded ring for the intersection product. In particular, $A_{G}^{*}(p t)$ is a graded ring.

For arbitrary $X$, the projection $X \times U \rightarrow U$ descends to a flat map

$$
p_{X}: X \times_{G} U \rightarrow U / G .
$$

Therefore, $A_{*}^{G}(X)$ is a graded $A_{G}^{*}(p t)$-module. The image of $p_{X}$ is the smooth variety $U / G$, and the fibers are isomorphic to $X$. Moreover, any two points in $U / G$ can be joined by a chain of rational curves (because the same holds in $U$, an open subset of a linear space). Thus, pull-back to a fiber is a welldefined map $A_{*}^{G}(X) \rightarrow A_{*}(X)$ invariant under the action of $A_{G}^{*}(p t)$. So we obtain a map

$$
\rho: A_{*}^{G}(X) /\left(A_{G}^{+}(p t)\right) \rightarrow A_{*}(X) .
$$


Any $G$-invariant subvariety $Y \subset X$ defines an equivariant class $[Y]$ in $A_{*}^{G}(X)$ : indeed, set

$$
[Y]:=\left[Y \times_{G} U\right] \in A_{*}\left(X \times_{G} U\right)
$$

for $U$ as above such that $\operatorname{codim}_{V}(V \backslash U)>\operatorname{codim}_{X}(Y)$. The image of $[Y]$ in $A_{*}(X)$ is the (usual) class of $Y$. In fact, we have a degree doubling cycle map

$$
c l_{X}^{G}: A_{*}^{G}(X) \rightarrow H_{*}^{G}(X, \mathbb{Z})
$$

to equivariant Borel-Moore homology [22]. For $X$ smooth, we have a cycle map

$$
c l_{X}^{G}: A_{G}^{*}(X) \rightarrow H_{G}^{*}(X, \mathbb{Z})
$$

to equivariant cohomology, which lifts the usual cycle map $c l_{X}$.

If $G$ acts on $X$ with a quotient $X \rightarrow X / G$ which is a principal $G$-bundle, then we have a smooth map $X \times_{G} V \rightarrow X / G$ with fibers isomorphic to $V$. It follows that

$$
A_{n-\operatorname{dim}(G)+\operatorname{dim}(U)}\left(X \times_{G} U\right)=A_{n-\operatorname{dim}(G)+\operatorname{dim}(V)}\left(X \times_{G} V\right)=A_{n-\operatorname{dim}(G)}(X / G) .
$$

Thus, $A_{*}^{G}(X)$ is isomorphic to $A_{*}(X / G)$ (with degree shifted by $\operatorname{dim}(G)$ ). A much deeper result is due to Edidin and Graham: they proved that $A_{*}^{G}(X)$ is isomorphic to $A_{*}(X / G)$ over $\mathbb{Q}$, whenever $G$ acts on $X$ with finite isotropy groups [22].

Let $X$ be a $G$-scheme and let $H \subset G$ be a closed subgroup. Then, for $U$ as above, the quotient $U \rightarrow U / H$ exists and is a principal $H$-bundle. Thus, there is a smooth map $X \times_{H} U \rightarrow X \times_{G} U$ with fiber $G / H$, which induces a map

$$
A_{*}^{G}(X) \rightarrow A_{*}^{H}(X)
$$

of degree $\operatorname{dim}(G / H)$. If moreover $H$ is a Levi subgroup of $G$, then $G / H$ is the unipotent radical of $G$ and hence is isomorphic to affine space. Thus, $A_{*}^{G}(X)$ is isomorphic to $A_{*}^{H}(X)$.

Our latter remark reduces many questions on equivariant Chow groups to the case of reductive groups. From now on, we assume that $G$ is reductive and connected; we denote by $T \subset G$ a maximal torus, by $W$ its Weyl group and by $B$ a Borel subgroup of $G$ containing $T$. Let $S_{\mathbb{Z}}$ (resp. $S$ ) be the symmetric algebra over the integers (resp. over the rationals) of the character group $\Xi(T) \simeq \Xi(B)$. Then we have the following analogue of Proposition 1, due to Edidin and Graham 21, 22. 
Theorem 10 Notation being as above, the graded ring $A_{T}^{*}(p t)$ is isomorphic to $S_{\mathbb{Z}}$. Moreover, the map $A_{G}^{*}(p t) \rightarrow A_{T}^{*}(p t)$ is injective over $\mathbb{Q}$ and identifies $A_{G}^{*}(p t)_{\mathbb{Q}}$ to $S^{W}$. Finally, for any $G$-scheme $X$, we have isomorphisms $A_{*}^{G}(X)_{\mathbb{Q}} \simeq A_{*}^{T}(X)_{\mathbb{Q}}^{W}$ and $S \otimes_{S^{W}} A_{*}^{G}(X)_{\mathbb{Q}} \simeq A_{*}^{T}(X)_{\mathbb{Q}}$.

Indeed, for any $\chi \in \Xi(T)$, consider the line bundle $L(\chi)=U \times_{T} \mathbb{C} \chi$ on $U / T$ as in Example 2 of Section 1. The first Chern class of $L(\chi)$ defines an element $c(\chi)$ of $A_{T}^{1}(p t)$, and the assignement $\chi \mapsto c(\chi)$ extends to the characteristic homomorphism $c: S_{\mathbb{Z}} \rightarrow A_{T}^{*}(p t)$. Using an explicit description of $U \rightarrow U / T$ as in Example 1 of Section 1, we see that $c$ is an isomorphism.

To prove the other statements, one begins by observing that the map

$$
X \times_{T} U \rightarrow X \times_{G} U
$$

factors through $X \times_{B} U$. Moreover, the map

$$
X \times_{T} U \rightarrow X \times_{B} U
$$

is smooth with fiber $B / T$ isomorphic to affine space, so it induces an isomorphism of Chow groups. Finally, the map

$$
X \times{ }_{B} U \rightarrow X \times_{G} U
$$

is smooth and proper with fiber the flag variety $G / B$. Then an analogue of the Leray-Hirsch theorem holds for this map [21], and we conclude as in the proof of Proposition 1 .

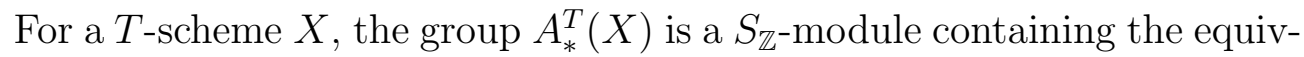
ariant classes $[Y]$ where $Y \subset X$ is a $T$-stable subvariety. If moreover $f$ is a rational function on $Y$ which is an eigenvector of $T$ of weight $\chi$, then the support of its divisor is $T$-invariant, and thus we can define a class $\operatorname{div}(f)$ in $A_{*}^{T}(X)$. In fact, we have

$$
\operatorname{div}(f)=\chi[Y]
$$

Indeed, $f$ can be seen as a rational section of the pull-back of the line bundle $L(\chi)=U \times_{T} \mathbb{C} \chi$ to $U \times_{T} Y$. Thus, $\operatorname{div}(f)$ represents the pull-back of $c(\chi)$ evaluated on the class of $U \times_{T} Y$, that is, $\chi[Y]$.

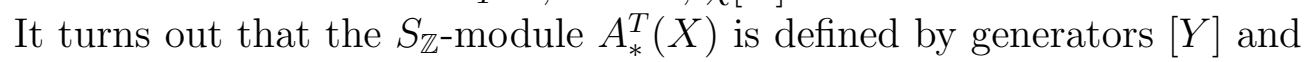
relations $[\operatorname{div}(f)]-\chi[Y]$ as above [14 2.1. The proof is based on an explicit construction of $U$ and $U / T$ as toric varieties, combined with a result 
of Fulton, MacPherson, Sottile and Sturmfels: For any scheme $X$ with an action of a connected solvable linear algebraic group $\Gamma$, the usual Chow group $A_{*}(X)$ is defined by generators $[Y]$ (where $Y \subset X$ is a $\Gamma$-stable subvariety) and relations $[\operatorname{div}(f)]$ (where $f$ is a rational function, eigenvector of $\Gamma$, on $Y$ as before) 25]. As a consequence, we obtain the following analogue of Proposition 2, valid for equivariant Chow groups of arbitrary schemes [14] 2.3 .

Proposition 11 Let $G$ be a connected reductive algebraic group, $T \subset G$ a maximal torus, and $X$ a $G$-scheme. Then the maps

$$
\rho_{T}: A_{*}^{T}(X) /\left(S_{\mathbb{Z},+}\right) \rightarrow A_{*}(X)
$$

and

$$
\rho_{G}: A_{*}^{G}(X)_{\mathbb{Q}} /\left(S_{+}^{W}\right) \rightarrow A_{*}(X)_{\mathbb{Q}}
$$

are isomorphisms.

Proof The first isomorphism follows immediatly from the results above; it can also be proved directly, as follows. Fixing a degree $n$ and using the isomorphism $A_{n}(X) \simeq A_{n+\operatorname{dim}(U)}(X \times U)$, we reduce to checking that the pull-back map

$$
A_{*}((X \times U) / T) /\left(S_{\mathbb{Z},+}\right) \rightarrow A_{*}(X \times U)
$$

is an isomorphism. But this follows from the argument of Example 2 above, where it was shown that $A_{*}(G / T) /\left(S_{\mathbb{Z},+}\right) \rightarrow A_{*}(G)$ is an isomorphism.

The second isomorphism follows from the first one, combined with the isomorphism $S \otimes_{S^{W}} A_{*}^{G}(X)_{\mathbb{Q}} \rightarrow A_{*}^{T}(X)_{\mathbb{Q}}$ of Theorem 10.

Consider for example $X=G$ where $G$ acts by left multiplication. Then $A_{T}^{*}(G)=A^{*}(G / T)=A^{*}(G / B)$ and we recover the structure of $A^{*}(G)$ given in Example 2 above. For $X=G / B$, we have $A_{G}^{*}(X)_{\mathbb{Q}}=S$ and we recover the description of $A^{*}(G / B)_{\mathbb{Q}}$ obtained earlier by comparing with cohomology.

More generally, let us apply this theory to a description of rational Chow rings of homogeneous spaces $G / H$ where $G$ is a connected linear algebraic group and $H \subset G$ is a closed connected subgroup. As above, we may assume that $G$ and $H$ are reductive.

Let $T_{H}$ be a maximal torus of $H$ with Weyl group $W_{H}$, and let $S_{H}$ be the symmetric algebra over $\mathbb{Q}$ of the character group of $T_{H}$. Finally, let $T$ be a maximal torus of $G$ containing $T_{H}$ and let $W$ be the Weyl group of 
$T$; then we have a restriction map $S \rightarrow S_{H}$. Observe that this maps sends $S^{W}$ to $S_{H}^{W_{H}}$. Indeed, the complexified space $S_{\mathbb{C}}$ can be identified to the algebra of polynomial functions on $\mathfrak{t}$, and the subspace $S_{\mathbb{C}}^{W}$ is then spanned by restrictions to $\mathfrak{t}$ of characters of $\mathfrak{g}$-modules [11] VIII.3.3. The latter restrict to characters of $\mathfrak{h}$-modules, which are $W_{H}$-invariants. We denote by $\left(S_{+}^{W}\right)$ the ideal of $S_{H}^{W_{H}}$ generated by restrictions of homogeneous elements of positive degree of $S^{W}$.

Corollary 12 1) Notation being as above, we have isomorphisms of graded rings

$$
A^{*}(G / H)_{\mathbb{Q}} \simeq S_{H}^{W_{H}} /\left(S_{+}^{W}\right)
$$

and

$$
A_{T}^{*}(G / H)_{\mathbb{Q}} \simeq S \otimes_{S^{W}} S_{H}^{W_{H}} .
$$

2) The following conditions are equivalent:

(i) The rational cohomology of $G / H$ vanishes in odd degree.

(ii) The ranks of $G$ and $H$ are equal.

(iii) The cycle map $c l_{G / H}: A^{*}(G / H)_{\mathbb{Q}} \rightarrow H^{*}(G / H)$ is an isomorphism.

Proof 1) For the first isomorphism, observe that $A_{G}^{*}(G / H)=A_{H}^{*}(p t)$ and apply Proposition 11. For the second one, recall that $A_{T}^{*}(G / H)_{\mathbb{Q}}$ is isomorphic to $S \otimes_{S^{W}} A_{G}^{*}(G / H)_{\mathbb{Q}}$.

2) (i) $\Rightarrow$ (ii) If $r k(H)<r k(G)$ then $T$ acts on $G / H$ without fixed point. It follows that the topological Euler characteristic of $G / H$ is zero. Thus, $G / H$ must have non trivial odd cohomology.

(ii) $\Rightarrow$ (iii) By assumption, we have $T_{H}=T$ and therefore,

$$
H^{*}(G / H)=H_{H}^{*}(G)=H_{T}^{*}(G)^{W_{H}}=H^{*}(G / T)^{W_{H}}
$$

with similar isomorphisms for (equivariant) Chow rings. Now the cycle map

$$
A^{*}(G / T)=A^{*}(G / B) \rightarrow H^{*}(G / B)=H^{*}(G / T)
$$

is a $W$-equivariant isomorphism. This implies our statement.

(iii) $\Rightarrow(\mathrm{i})$ is obvious.

Recall that any homogeneous space $G / H$ under a connected linear algebraic group has the structure of a vector bundle over the homogeneous space $G_{c} / H_{c}$ where $H_{c}$ is a maximal compact subgroup of $H$, and $G_{c} \supset H_{c}$ is a 
maximal compact subgroup of $G$. In particular, $G / H$ has the same cohomology as $G_{c} / H_{c}$. The cohomology ring of the latter has been intensively studied, see e.g. [8]; its structure is in general more complicated than that of $A^{*}(G / H)_{\mathbb{Q}}$. Concerning the integral Chow ring $A^{*}(G / H)$, we are led to the following

Question 1. How to describe torsion in Chow rings of homogeneous spaces?

As seen in Example 2 above, the Chow ring of a connnected linear algebraic group is torsion-free (that is, isomorphic to $\mathbb{Z}$ ) if and only if the characteristic homomorphism $c: S_{\mathbb{Z}} \rightarrow A^{*}(G / B)$ is surjective, that is, the group is special in the sense of [28].

Question 2. Notation being as above, when is $A^{*}(G / H)$ finite in positive degree? Equivalently, when is the restriction map $S^{W} \rightarrow S_{H}^{W_{H}}$ surjective? Or, denoting by $\mathbb{C}[\mathfrak{g}]$ the ring of polynomial functions on $\mathfrak{g}$ and using the Chevalley restriction theorem [11] VIII.3.3: when is the restriction map $\mathbb{C}[\mathfrak{g}]^{G} \rightarrow \mathbb{C}[\mathfrak{h}]^{H}$ surjective?

This holds e.g. when $H$ is isomorphic to SL(2) or to PSL(2): indeed, the Killing form of $\mathfrak{g}$ restricts then to a non zero quadratic element of $\mathbb{C}[\mathfrak{h}]^{H}$, which generates this algebra. But this does not hold if $H$ is a proper subgroup of maximal rank of $G$, because we then have $T_{H}=T$ and $W_{H} \neq W$. It turns out that this does not hold for certain pairs of semisimple groups $(G, H)$ as well. The following example was pointed out by Bram Broer: let $G$ be semisimple of type $E_{6}$ and $H \subset G$ be a maximal semisimple subgroup of a parabolic subgroup of type $D_{5}$. Then the sequence of degrees of a minimal system of homogeneous generators for $S^{W}$ (resp. $S_{H}^{W_{H}}$ ) is $(2,5,6,8,9,12)$ (resp. $(2,4,5,6,8))$ : a generator of degree 4 of $S_{H}^{W_{H}}$ cannot be in the image of $S^{W}$.

\section{Localization and equivariant multiplicities}

As in the previous section, we consider schemes over the field of complex numbers. If $T$ is a torus and $X$ a $T$-scheme, we denote by

$$
i_{T}: X^{T} \rightarrow X
$$


the inclusion of the fixed point subscheme. Then $i_{T}$ induces a homomorphism of equivariant Chow groups

$$
i_{T *}: A_{*}^{T}\left(X^{T}\right) \rightarrow A_{*}^{T}\left(X^{T}\right)
$$

which is $S_{\mathbb{Z}}$-linear (here $S_{\mathbb{Z}}$ denotes as before the symmetric algebra over the integers of the character group of $T$ ). Moreover, we have a natural isomorphism

$$
A_{*}^{T}\left(X^{T}\right) \simeq S_{\mathbb{Z}} \otimes A_{*}\left(X^{T}\right) .
$$

Indeed, for fixed degree $n$, we have $A_{n}^{T}\left(X^{T}\right)=A_{n}\left(X^{T} \times U / T\right)$ where $U$ is an open $T$-invariant subset of a $T$-module, such that the quotient $U \rightarrow U / T$ exists and is a principal $T$-bundle, and that the codimension of $V \backslash U$ is large enough. Moreover, as in the second example of Section 1, we can find $U$ such that $U / T$ is a product of projective spaces. It follows that $A_{*}\left(X^{T} \times U / T\right)=A_{*}\left(X^{T}\right) \otimes A_{*}(U / T)$ [24] Chapter 3 (note that the analogue of the Künneth formula does not hold for Chow groups of products of arbitrary schemes).

We can now state a version of the localization theorem, due to Edidin and Graham [23] in the more general setting of higher equivariant Chow groups.

Theorem 13 For any $T$-scheme $X$, the $S_{\mathbb{Z}}$-linear map

$$
i_{T *}: A_{*}^{T}\left(X^{T}\right) \rightarrow A_{*}^{T}(X)
$$

becomes an isomorphism after inverting finitely many non trivial characters.

Proof (after [14 2.3) By assumption, $X$ is a finite union of $T$-stable affine open subsets $X_{i}$. The ideal of each fixed point subscheme $X_{i}^{T}$ is generated by all regular functions on $X_{i}$ which are eigenvectors of $T$ with a non trivial weight. We can choose a finite set of such generators $\left(f_{i j}\right)$, with respective weights $\chi_{i j}$.

Let $Y \subset X$ be a $T$-invariant subvariety. If $Y$ is not fixed pointwise by $T$, then one of the $f_{i j}$ defines a non zero rational function on $Y$. Thus, we can write

$$
[Y]=\chi_{i j}^{-1} \operatorname{div}\left(f_{i j}\right)
$$

in $A_{*}^{T}(X)\left[1 / \chi_{i j}\right]$. It follows by induction that $i_{T *}$ is surjective after inverting the $\chi_{i j}$ 's.

For injectivity, we may assume that $X$ is not fixed pointwise by $T$. Let $Y$ be an irreducible component of $X$ not contained in $X^{T}$. Choose $f_{i j}$ as 
before; denote by $|D|$ the union of the support of the divisor of $f_{i j}$ in $Y$, and of the irreducible components of $X$ which are not equal to $Y$. Then $|D|$ contains all fixed points of $X$. Denote by $j:|D| \rightarrow X$ the inclusion. Let $U \rightarrow U / T$ be as in the definition of equivariant Chow groups, and let $L\left(\chi_{i j}\right)$ be the line bundle on $U / T$ associated with the character $\chi_{i j}$. Denote by $p_{X}: X \times_{T} U \rightarrow U / T$ the projection. Then we have a pseudo-divisor on $X \times_{T} U$ [24] 2.2:

$$
\left(p_{X}^{*} L\left(\chi_{i j}\right),|D| \times_{T} U, f_{i j}\right)
$$

which defines a homogeneous map of degree -1

$$
j^{*}: A_{*}^{T}(X) \rightarrow A_{*}^{T}(|D|)
$$

such that composition $j^{*} \circ j_{*}$ is multiplication by $\chi_{i j}$. Thus, the map

$$
j_{*}: A_{*}^{T}(|D|) \rightarrow A_{*}^{T}(X)
$$

is injective after inverting $\chi_{i j}$. We conclude by Noetherian induction.

If moreover $X$ is smooth, then the fixed point scheme $X^{T}$ is smooth, too. Then, as in the end of Section 1, we see that the analogue of Theorem 3 holds in the algebraic setting. We refer to [23] for applications to the Bott residue formula [10].

Any projective smooth algebraic $T$-variety $X$ admits a $T$-stable cellular decomposition, by a result of Bialynicki-Birula [6]. It follows easily that the cycle maps $c l_{X}^{T}: A_{T}^{*}(X) \rightarrow H_{T}^{*}(X, \mathbb{Z})$ and $c l_{X}: A^{*}(X) \rightarrow H^{*}(X, \mathbb{Z})$

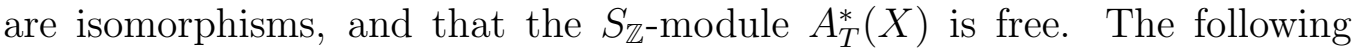
extension of this result is proved in [14] 3.2 .

Theorem 14 For any projective smooth $T$-variety $X$, the $S$-module $A_{T}^{*}(X)_{\mathbb{Q}}$ is free. Furthermore, the pull-back map

$$
i_{T}^{*}: A_{T}^{*}(X)_{\mathbb{Q}} \rightarrow A_{T}^{*}\left(X^{T}\right)_{\mathbb{Q}}
$$

is injective, and becomes surjective after inverting finitely many non trivial characters of $T$. If moreover the cycle map

$$
c l_{X^{T}}: A^{*}\left(X^{T}\right)_{\mathbb{Q}} \rightarrow H^{*}\left(X^{T}, \mathbb{Q}\right)
$$

is an isomorphism, then both cycle maps

$$
c l_{X}^{T}: A_{T}^{*}(X)_{\mathbb{Q}} \rightarrow H_{T}^{*}(X), c l_{X}: A^{*}(X)_{\mathbb{Q}} \rightarrow H^{*}(X)
$$

are isomorphisms, too. 
It follows that the analogue of Theorem 6 holds in this setting, with the same proof.

Denote by $Q$ the quotient field of $S_{\mathbb{Z}}$; then $Q$ is the field of rational functions in $\operatorname{dim}(T)$ variables, with rational coefficients.

Corollary 15 Let $X$ be an equidimensional $T$-scheme with finite fixed point set. Then we have in $A_{*}^{T}(X) \otimes_{S_{\mathbb{Z}}} Q \simeq A_{*}^{T}\left(X^{T}\right) \otimes_{S_{\mathbb{Z}}} Q \simeq \oplus_{x \in X^{T}} Q[x]$ :

$$
[X]=\sum_{x \in X^{T}}\left(e_{x} X\right)[x]
$$

for uniquely defined coefficients $e_{x} X \in Q$ which are homogeneous of degree $-\operatorname{dim}(X)$. If moreover $X$ is smooth at $x$, then

$$
e_{x} X=\frac{1}{\operatorname{det}\left(T_{x} X\right)} .
$$

Proof The first assertion follows from Theorem 13. For the second one, we may replace $X$ by an open $T$-stable neighborhood of $x$, and thus assume that $X$ is smooth. Denote by $i_{x}$ the inclusion of $x$ into $X$. Then we know that $i_{x}^{*} \circ i_{x *}$ is multiplication by the determinant of the action of $T$ in $T_{x} X$. Comparing coefficients of $[x]$ in $i_{x}^{*}[X]=[x]$, we obtain our result.

The homogeneous rational function $e_{x} X$ is called the equivariant multiplicity of $X$ at $x$. The following observation allows to compute it in many examples.

Lemma 16 Let $X, Y$ be $T$-varieties and let $\pi: Y \rightarrow X$ be a proper surjective equivariant morphism of finite degree d. If $Y^{T}$ is finite, then we have for any $x \in X^{T}$ :

$$
e_{x} X=\frac{1}{d} \sum_{y \in Y^{T}, \pi(y)=x} e_{y} Y .
$$

This follows immediately from equality $\pi_{*}[Y]=d[X]$ in $A_{*}^{T}(X)$. In particular, if $\pi$ is a resolution of singularities (that is, $Y$ is smooth and $\pi$ is birational), then

$$
e_{x} X=\sum_{y \in Y^{T}, \pi(y)=x} \frac{1}{\operatorname{det}\left(T_{y} Y\right)} .
$$


Example (Schubert varieties)

Recall that the $T$-fixed points in the flag variety $G / B$ are the $w B$ for $w \in W$. Moreover, the $T$-fixed points in the Schubert variety

$$
X(w):=\overline{B w B} / B
$$

are the $x B$ where $x \in W$ and $x \leq w$ for the Bruhat ordering. Because the map $w \mapsto w B$ is injective, we will write $w$ for $w B$, etc. Let us determine $e_{x} X(w)$

Denote by $\Phi$ the root system of $(G, T)$, by $\Phi^{+}$the subset of positive roots such that the roots of $(B, T)$ are negative, and by $\Pi$ the corresponding set of simple roots. Then the set of weights of $T$ in the tangent space $T_{w} G / B$ is $w\left(\Phi^{+}\right)$and each such weight has multiplicity one. We thus have

$$
e_{w} G / B=\prod_{\alpha \in w\left(\Phi^{+}\right)} \alpha^{-1} .
$$

Moreover, $w$ is a smooth point of $X(w)$ and the set of weights of $T_{w} X(w)$ is $\Phi^{-} \cap w\left(\Phi^{+}\right)$, whence

$$
e_{w} X(w)=\prod_{\alpha \in \Phi^{-} \cap w\left(\Phi^{+}\right)} \alpha^{-1}
$$

To obtain $e_{x} X(w)$ for arbitrary $x$, we use the Bott-Samelson resolution of singularities of $X(w)$, which we recall briefly. For any $\alpha \in \Pi$, denote by $s_{\alpha}$ the corresponding reflection and by $P_{\alpha}=B \cup B s_{\alpha} B$ the corresponding minimal parabolic subgroup. We can find $\alpha \in \Pi$ and $\tau \in W$ such that $w=s_{\alpha} \tau$ and that $\ell(w)=\ell(\tau)+1$ (here $\ell$ denotes the length function on $W$ ). Then $X(w)=P_{\alpha} X(\tau)$ and the map

$$
\pi: P_{\alpha} \times_{B} X(\tau) \rightarrow X(w)
$$

is birational. Iterating this construction, we obtain a $B$-equivariant birational map

$$
P_{\alpha_{1}} \times_{B} P_{\alpha_{2}} \times_{B} \cdots \times_{B} P_{\alpha_{n}} / B \rightarrow X(w)
$$

where $w=s_{\alpha_{1}} s_{\alpha_{2}} \cdots s_{\alpha_{n}}$ is a reduced decomposition. Moreover, the variety on the left is projective, smooth and contains only finitely many $T$-fixed points: the classes of sequences $\left(s_{1}, \ldots, s_{n}\right)$ where each $s_{j}$ is either $s_{\alpha_{j}}$ or 1 . 
We claim that

$$
e_{x} X(w)=\frac{e_{x} X(\tau)-s_{\alpha}\left(e_{s_{\alpha} x} X(\tau)\right)}{\alpha},
$$

which determines inductively equivariant multiplicities of Schubert varieties. Indeed, the $T$-fixed points in the fiber $\pi^{-1}(x)$ are the classes $(1, x) B$ and $\left(s_{\alpha}, s_{\alpha} x\right) B$, where the first (resp. second) point occurs if and only if $x \leq \tau$ (resp. $\left.s_{\alpha} x \leq \tau\right)$. Finally, $(1, x) B$ has a $T$-invariant neighborhood isomorphic to $\mathbb{C}(\alpha) \times X(\tau)$ where $\mathbb{C}(\alpha)$ is the one-dimensional $T$-module with weight $\alpha$. It follows that

$$
e_{(1, x) B} P_{\alpha} \times{ }_{B} X(\tau)=\frac{e_{x} X(\tau)}{\alpha}
$$

and that a similar equality holds for $e_{\left(s_{\alpha}, s_{\alpha} x\right) B} P_{\alpha} \times_{B} X(\tau)$. Applying Lemma 16, we obtain our claim.

Let now

$$
w=s_{\alpha_{1}} \cdots s_{\alpha_{n}}
$$

be a reduced decomposition. Applying repeatedly the formula above (or equivalently, using the full Bott-Samelson resolution), we obtain an explicit expression for $e_{x} X(w)$ [3] Proposition 3.3.1 and 445]:

$$
e_{x} X(w)=\sum_{s_{1}, \ldots, s_{n}} \prod_{j=1}^{n} s_{1} \cdots s_{j}\left(\alpha_{j}^{-1}\right)
$$

where the sum runs over all sequences $\left(s_{1}, \ldots, s_{n}\right)$ such that $s_{j}=s_{\alpha_{j}}$ or 1 , and that $s_{1} \cdots s_{n}=x$ (such a sequence is called a subexpression of the reduced expression for $w$ ).

Consider for example $w=s_{\alpha} s_{\beta}$ where $\alpha$ and $\beta$ are distinct simple roots. Then we obtain

$$
e_{1} X\left(s_{\alpha} s_{\beta}\right)=-e_{s_{\beta}} X\left(s_{\alpha} s_{\beta}\right)=\frac{1}{\alpha \beta}, e_{s_{\alpha} s_{\beta}} X\left(s_{\alpha} s_{\beta}\right)=-e_{s_{\alpha}} X\left(s_{\alpha} s_{\beta}\right)=\frac{1}{\alpha s_{\alpha}(\beta)} .
$$

If moreover $\alpha$ and $\beta$ are connected in the Dynkin diagram, then $s_{\alpha} s_{\beta} s_{\alpha}$ has length 3 , and we obtain

$$
e_{1} X\left(s_{\alpha} s_{\beta} s_{\alpha}\right)=-e_{s_{\alpha}} X\left(s_{\alpha} s_{\beta} s_{\alpha}\right)=\frac{-\left\langle\beta, \alpha^{\vee}\right\rangle}{\alpha \beta s_{\alpha}(\beta)}
$$

which shows that $e_{x} X(w)$ is not always the inverse of a product of roots. 
To understand better $e_{x} X(w)$, we recall the construction of a slice $\mathcal{N}_{x, w}$ to the orbit $B x$ in $X(w)$. Denote by $U, U_{x}$ (resp. $U^{-}, U_{x}^{-}$) the unipotent subgroup of $G$ normalized by $T$ with root set $\Phi^{-}, \Phi^{-} \cap x\left(\Phi^{+}\right)$(resp. $\Phi^{+}$, $\left.\Phi^{+} \cap x\left(\Phi^{+}\right)\right)$. Then, by the Bruhat decomposition, the set

$$
U^{-} x B / B:=U^{-} x
$$

is an open $T$-invariant neighborhood of $x$ in $G / B$, and the product map

$$
U_{x} \times U_{x}^{-} x \rightarrow U^{-} x,(u, \xi) \mapsto u \xi
$$

is an isomorphism, which restricts to an isomorphism $U_{x} \times x \rightarrow B x$. Thus,

$$
\mathcal{N}_{x, w}:=U_{x}^{-} \cap X(w)
$$

is a locally closed, $T$-stable subvariety of $X(w)$ containing $x$, and the product map

$$
U_{x} \times \mathcal{N}_{x, w} \rightarrow X(w)
$$

is an open immersion. It follows that

$$
e_{x} X(w)=\left(e_{x} U_{x}\right)\left(e_{x} \mathcal{N}_{x, w}\right)=\left(\prod_{\alpha \in \Phi^{-} \cap x\left(\Phi^{+}\right)} \alpha^{-1}\right) e_{x} \mathcal{N}_{x, w} .
$$

Moreover, $e_{x} \mathcal{N}_{x, w}$ is a rational function of degree $-\operatorname{dim} \mathcal{N}_{x, w}=l(x)-l(w)$.

Consider the simplest case where $l(w)=l(x)+1$. Choose a reduced decomposition $w=s_{\alpha_{1}} \cdots s_{\alpha_{n}}$. Then there exists an index $j$ such that

$$
x=s_{\alpha_{1}} \cdots \widehat{s_{\alpha_{j}}} \cdots s_{\alpha_{n}} .
$$

It follows that $w=s_{\beta} x$ where $\beta=s_{\alpha_{1}} \cdots s_{\alpha_{j-1}}\left(\alpha_{j}\right)$ is in $\Phi^{+} \cap x\left(\Phi^{+}\right)$. Now the explicit formula for $e_{x} X(w)$ gives immediately

$$
e_{x} X(w)=\left(\prod_{\alpha \in \Phi^{-} \cap x\left(\Phi^{+}\right)} \alpha^{-1}\right) \beta^{-1}, e_{x} \mathcal{N}_{x, w}=\beta^{-1} .
$$

In fact, the $T$-variety $\mathcal{N}_{x, w}$ is isomorphic to the module with weight $\beta$ (this will be checked at the end of Section 5).

For arbitrary $x$ and $w$, we are led to the following

Question. What is the structure of the $T$-variety $\mathcal{N}_{x, w}$ ? 
This is especially interesting in the case where $X(x)$ is an irreducible component of the singular locus of $X(w)$; equivalently, $x$ is an isolated singular point of $\mathcal{N}_{x, w}$. Then the singularity of $\mathcal{N}_{x, w}$ at $x$ is the generic singularity of $X(w)$ along $B x$.

Little seems to be known about this question. Here are two partial results: In the case where $l(w)=l(x)+2$, it can be shown that $\mathcal{N}_{x, w}$ is a toric surface for a quotient of $T$ (see the end of Section 5), and thus, a cone over the projective line. On the other hand, generic singularities of Schubert varieties in $G / P$ are described in [15] when $P \supset B$ is a maximal parabolic subgroup associated with a minuscule or cominuscule fundamental weight. These singularities turn out to be multicones over homogeneous spaces.

Back to the general case of a torus action with isolated fixed points, let us give an interpretation of equivariant multiplicity for an attractive fixed point, in the following sense.

Definition. A fixed point $x$ is attractive if all weights of $T$ in the tangent space $T_{x} X$ are contained in some open half-space, that is, some oneparameter subgroup of $T$ acts on $T_{x} X$ with positive weights only.

Equivalently, all $T$-orbits in some open $T$-stable neighborhood of $x$ contain that point in their closure. In fact, the set

$$
X_{x}=:\{\xi \in X \mid x \in \overline{T \xi}\}
$$

is the unique open affine $T$-stable neighborhood of $x$ in $X$; clearly, $x$ is the unique closed $T$-orbit in $X_{x}$.

For attractive $x$, we denote by $\chi_{1}, \ldots, \chi_{n}$ the weights of $T$ in $T_{x} X$. Let $\Xi_{*}(T)$ be the lattice of one-parameter subgroups of $T$, and $\Xi_{*}(T)_{\mathbb{R}}$ the associated real vector space. Set

$$
\sigma_{x}:=\left\{\lambda \in \Xi_{*}(T)_{\mathbb{R}} \mid\left\langle\lambda, \chi_{i}\right\rangle \geq 0 \text { for } 1 \leq i \leq n\right\}
$$

Then $\sigma_{x}$ is a rational polyhedral convex cone in $\Xi_{*}(T)_{\mathbb{R}}$ with a non empty interior $\sigma_{x}^{0}$. Any $\lambda \in \sigma_{x}^{0} \cap \Xi_{*}(T)$ defines a grading of the algebra of regular functions $\mathbb{C}\left[X_{x}\right]$, by setting

$$
\mathbb{C}\left[X_{x}\right]_{n}:=\bigoplus_{\chi \in \Xi(T),\langle\chi, \lambda\rangle=n} \mathbb{C}\left[X_{x}\right]_{\chi}
$$


Let $d$ be the dimension of $X$. Then there exists a positive rational number $e$ such that

$$
\sum_{m=0}^{n} \operatorname{dim} \mathbb{C}\left[X_{x}\right]_{m}=e \frac{n^{d}}{d !}+o\left(n^{d}\right) .
$$

This number is called the multiplicity of $\mathbb{C}\left[X_{x}\right]$ for the grading defined by $\lambda$. It is easily seen that $e$ is the value at $\lambda$ of $e_{x} X$ (viewed as a rational function on $\left.\Xi_{*}(T)_{\mathbb{R}}\right)$, and that the product $\chi_{1} \cdots \chi_{n}=\operatorname{det}\left(T_{x} X\right)$ is a denominator for $e_{x} X$ 14 4.4. The homogeneous polynomial function

$$
\chi_{1} \cdots \chi_{n} e_{x} X=\operatorname{det}\left(T_{x} X\right) e_{x} X=: J_{x} X
$$

of degree $n-\operatorname{dim}(X)$ is the Joseph polynomial introduced in 34 in relation to representation theory, see also [9].

In the example of the flag manifold, each fixed point $x$ is attractive, and $(G / B)_{x}=x U^{-} B / B$ where $U^{-} \subset G$ is the unipotent subgroup normalized by $T$ with root set $\Phi^{+}$. Moreover, the cone $\sigma_{x}$ is the image of the positive Weyl chamber under $x^{-1} \in W$. Because $\mathcal{N}_{x, w}$ is contained in $U_{x}^{-}$, the product of all roots in $\Phi^{+} \cap x\left(\Phi^{+}\right)$is a denominator for $e_{x} \mathcal{N}_{x, w}$. A more precise result will be given at the end of Section 5 .

On the other hand, if $X$ is a toric variety, then any fixed point $x \in X$ is attractive, and $\sigma_{x}$ is the cone associated with the affine toric variety $X_{x}$. Moreover, for any $\lambda \in \sigma_{x}^{0}$, the value at $\lambda$ of $e_{x} X$ is $d$ ! times the volume of the convex polytope $P_{x}(\lambda):=\left\{x \in \Xi(T)_{\mathbb{R}} \mid\langle\mu, x\rangle \geq 0 \forall \mu \in \sigma_{x},\langle\lambda, x\rangle \leq 1\right\}$ (see [14 5.2). Here the volume form on $\Xi(T)_{\mathbb{R}}$ is normalized so that the quotient by the lattice $\Xi(T)$ has volume 1 .
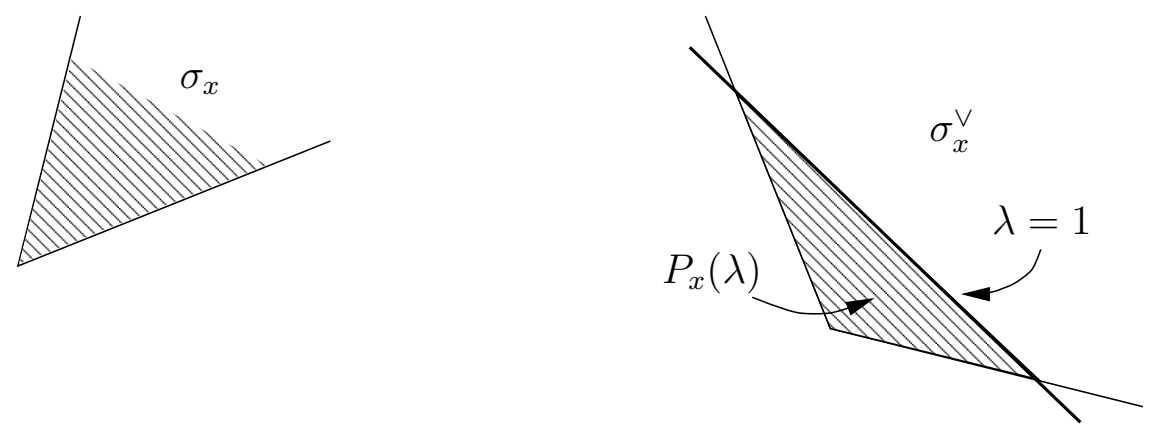


\section{Criteria for (rational) smoothness}

We begin by giving a smoothness criterion at an attractive fixed point of a torus action, in terms of fixed points of subtori of codimension 1 as in Theorem 6.

Theorem 17 For a $T$-scheme $X$ with an attractive fixed point $x$, the following conditions are equivalent:

(i) $X$ is smooth at $x$.

(ii) For any subtorus $T^{\prime} \subset T$ of codimension 1, the fixed point set $X^{T^{\prime}}$ is smooth at $x$, and we have

$$
e_{x} X=\prod_{T^{\prime}} e_{x}\left(X^{T^{\prime}}\right)
$$

(product over all subtori of codimension 1).

Proof Replacing $X$ by $X_{x}$, we may assume that $X$ is affine.

(i) $\Rightarrow$ (ii) By the graded Nakayama lemma, $X$ is equivariantly isomorphic to a $T$-module $V$. Then each $X^{T^{\prime}}$ is isomorphic to the $T$-module $V^{T^{\prime}}$; thus, $X^{T^{\prime}}$ is smooth. Moreover, we have by Corollary 15:

$$
e_{x} X=\prod \frac{1}{\chi^{\operatorname{dim}\left(V_{\chi}\right)}}
$$

(product over all characters $\chi$ of $T$, where $V_{\chi}$ denotes the corresponding eigenspace). Then

$$
e_{x}\left(X^{T^{\prime}}\right)=\prod_{\chi, \chi \mid T^{\prime}=0} \frac{1}{\chi^{\operatorname{dim}\left(V_{\chi}\right)}}
$$

for each subtorus $T^{\prime}$. Our formula follows.

(ii) $\Rightarrow$ (i) There exists an equivariant closed embedding

$$
\iota: X \rightarrow T_{x} X
$$

such that $\iota(x)=0$. For any subtorus $T^{\prime} \subset T$ of codimension one, we have a subspace $T_{x}\left(X^{T^{\prime}}\right)$ of $T_{x} X$. Let $V \subset T_{x} X$ be the span of all these subspaces; then $V$ is a $T$-submodule of $T_{x} X$. Choose an equivariant projection

$$
p: T_{x} X \rightarrow V
$$


and denote by

$$
\pi: X \rightarrow V
$$

the composition of $\iota$ and $p$. Then $\pi$ is equivariant, $\pi(x)=0$ and the differential of $\pi$ at $x$ induces isomorphisms $T_{x}\left(X^{T^{\prime}}\right) \rightarrow V^{T^{\prime}}$ for all subtori $T^{\prime} \subset T$ of codimension 1. Because $X^{T^{\prime}}$ is smooth at the attractive point $x$, it follows by the graded Nakayama lemma that $\pi$ restricts to isomorphisms $X^{T^{\prime}} \rightarrow V^{T^{\prime}}$.

We claim that the morphism $\pi$ is finite. By the graded Nakayama lemma again, it is enough to check that the set $\pi^{-1}(0)$ consists of $x$, or even that $\pi^{-1}(0)$ is finite (because $x$ is the unique fixed point of $X$ ). But if $\pi^{-1}(0)$ is infinite, then this closed $T$-stable subset of $X$ contains a $T$-stable closed curve $C$. So $C$ contains $x$ and is fixed pointwise by a subtorus $T^{\prime} \subset T$ of codimension 1. This contradicts the fact that restriction of $\pi$ to $X^{T^{\prime}}$ is injective.

Now observe that

$$
\operatorname{dim}(X)=-\operatorname{deg}\left(e_{x} X\right)=-\sum_{T^{\prime}} \operatorname{deg}\left(e_{x}\left(X^{T^{\prime}}\right)\right)=\sum_{T^{\prime}} \operatorname{dim}\left(X^{T^{\prime}}\right)=\operatorname{dim}(V) .
$$

Thus, the finite morphism $\pi: X \rightarrow V$ is surjective. Then the algebra of regular functions $\mathbb{C}[X]$ is a finite torsion-free module over $\mathbb{C}[V]$; let $d$ be its rank. We have

$$
e_{x} X=d e_{x} V=d \prod_{T^{\prime}} e_{x}\left(V^{T^{\prime}}\right)=d \prod_{T^{\prime}} e_{x}\left(X^{T^{\prime}}\right)=d e_{x} X
$$

and $e_{x} X$ is non zero, because no $e_{x}\left(X^{T^{\prime}}\right)$ is. Thus, $d=1$, that is, $\pi$ is birational. As $V$ is normal, it follows that $\pi$ is an isomorphism.

We now adapt these arguments to obtain a similar criterion for rational smoothness at an attractive fixed point. Recall the following

Definition [37 An algebraic variety $X$ is rationally smooth of dimension $n$ (or a rational cohomology manifold of dimension $n$ ) if we have $H^{m}(X, X \backslash x)=$ 0 for $m \neq n$, and $H^{n}(X, X \backslash x) \simeq \mathbb{Q}$, for all $x \in X$. A point $x \in X$ is rationally smooth if it admits a rationally smooth neighborhood.

By recent work of A. Arabia [4], an attractive fixed point $x$ such that all weights in $T_{x} X$ have multiplicity 1 is rationally smooth if and only if: a punctured neighborhood of $x$ is rationally smooth, and $e_{x} X$ is the inverse of a polynomial. Here is an extension of this result. 
Theorem 18 Let $X$ be a T-variety with an attractive fixed point $x$ such that a punctured neighborhood of $x$ in $X$ is rationally smooth. Then the following conditions are equivalent:

(i) The point $x$ is rationally smooth.

(ii) For any subtorus $T^{\prime} \subset T$ of codimension 1, the point $x$ is rationally smooth in $X^{T^{\prime}}$, and there exists a positive rational number $c$ such that

$$
e_{x} X=c \prod_{T^{\prime}} e_{x}\left(X^{T^{\prime}}\right)
$$

(product over all subtori of codimension 1). If moreover each $X^{T^{\prime}}$ is smooth, then $c$ is an integer.

(iii) For any subtorus $T^{\prime} \subset T$ of codimension 1, the point $x$ is rationally smooth in $X^{T^{\prime}}$, and we have $\operatorname{dim}(X)=\sum_{T^{\prime}} \operatorname{dim}\left(X^{T^{\prime}}\right)$ (sum over all subtori of codimension 1).

Proof We begin with some observations on the local structure of $X$ at $x$. First, we may assume that $X$ is affine. Then $x$ is the unique closed $T$-orbit in $X$, and the punctured space $\dot{X}:=X \backslash x$ is rationally smooth.

Choose a one-parameter subgroup $\lambda$ such that all weights of $\lambda$ in $T_{x} X$ are positive. For the action of $\mathbb{C}^{*}$ on $\dot{X}$ via $\lambda$, the quotient

$$
\dot{X} / \mathbb{C}^{*}=: \mathbb{P}(X)
$$

exists and is a projective variety. Indeed, let $\iota: X \rightarrow T_{x} X$ be an equivariant embedding such that $\iota(x)=0$. Then $\iota$ induces a closed embedding of $\mathbb{P}(X)$ into $\mathbb{P}\left(T_{x} X\right)$, and $\mathbb{P}\left(T_{x} X\right)$ is a weighted projective space.

Moreover, $\mathbb{P}(X)$ is rationally smooth. Indeed, $\dot{X}$ is covered by $\mathbb{C}^{*}$-stable open subsets of the form

$$
\mathbb{C}^{*} \times_{\Gamma} Y
$$

where $\Gamma$ is a finite subgroup of $\mathbb{C}^{*}$, and $Y$ is a $\Gamma$-stable subvariety of $\dot{X}$; then $\mathbb{P}(X)$ is covered by the quotients $Y / \Gamma$. Because $\dot{X}$ is rationally smooth, $\mathbb{C}^{*} \times Y$ and $Y$ are, too, and so are the quotients $Y / \Gamma$.

The action of $T$ on $X$ induces an action of $T / \lambda\left(\mathbb{C}^{*}\right)$ on $\mathbb{P}(X)$ for which the fixed point set is the disjoint union of the $\mathbb{P}\left(X^{T^{\prime}}\right)$. Indeed, $T$-fixed points in $\mathbb{P}(X)$ correspond to $T$-orbits of dimension 1 in $X$.

Observe that $x$ is rationally smooth of dimension $n$ if and only if $\dot{X}$ is a rational cohomology sphere of dimension $n-1$. Indeed, because the action of $\mathbb{C}^{*}$ on $X$ extends to a map $\mathbb{C} \times X \rightarrow X$ sending $0 \times X$ to 0 , the space $X$ 
is contractible. Thus, $H^{m}(X)=0$ for all $m>0$. Now our claim follows from the long exact sequence in relative cohomology.

Observe finally that $x$ is rationally smooth if and only if $\mathbb{P}(X)$ is a rational cohomology complex projective space $\mathbb{P}^{d-1}$ where $d=\operatorname{dim}(X)$. Indeed, the group $S^{1} \subset \mathbb{C}^{*}$ acts on $\dot{X}$ without fixed points, whence a Gysin exact sequence

$$
\cdots \rightarrow H^{m}(\dot{X}) \rightarrow H^{m-1}\left(\dot{X} / S^{1}\right) \rightarrow H^{m+1}\left(\dot{X} / S^{1}\right) \rightarrow H^{m+1}(\dot{X}) \rightarrow \cdots
$$

It follows that $\dot{X}$ is a rational cohomology $(n-1)$-sphere if and only if: $n$ is even and $\dot{X} / S^{1}$ has the rational cohomology of $\mathbb{P}^{(n-1) / 2}$. But the map

$$
\dot{X} / S^{1} \rightarrow \dot{X} / \mathbb{C}^{*}=\mathbb{P}(X)
$$

induces an isomorphism in rational cohomology. Moreover, because $\mathbb{P}(X)$ is rationally smooth and projective of dimension $d-1$, the maximal degree occuring in its rational cohomology is $2(d-1)$ and this forces $n=2 d$.

(i) $\Rightarrow$ (ii) The first assertion follows from the discussion above, together with a theorem of Smith: the fixed point set of a torus acting on a rational cohomology sphere is a rational cohomology sphere as well [31] Theorem IV.2.

For each $T^{\prime}$, choose a homogeneous system of parameters of the algebra $\mathbb{C}\left[X^{T^{\prime}}\right]$ (graded by the action of $T / T^{\prime} \simeq \mathbb{C}^{*}$ ). This defines a $T$-equivariant finite surjective morphism

$$
\pi_{T^{\prime}}: X^{T^{\prime}} \rightarrow V^{T^{\prime}}
$$

where $V^{T^{\prime}}$ is a $T$-module with trivial action of $T^{\prime}$. We can extend $\pi_{T^{\prime}}$ to an equivariant morphism

$$
X \rightarrow V^{T^{\prime}}
$$

still denoted by $\pi_{T^{\prime}}$. Thus, we obtain an equivariant morphism

$$
\pi: X \rightarrow V
$$

where $V$ is the direct sum of the $V^{T^{\prime}}$ over all $T^{\prime}$ (observe that only finitely many such spaces are non zero). Moreover, the morphism $\pi$ is finite. Indeed, it restricts to a finite morphism on each $X^{T^{\prime}}$, and thus $\pi^{-1}(0)$ contains no $T$-stable curve by the argument of Theorem 17. 
Because $\mathbb{P}(X)$ and all $\mathbb{P}\left(X^{T^{\prime}}\right)$ are rational cohomology projective spaces, we have

$$
\begin{aligned}
\operatorname{dim}(X)=\chi(\mathbb{P}(X))=\chi\left(\mathbb{P}(X)^{T}\right) & =\sum_{T^{\prime}} \chi\left(\mathbb{P}\left(X^{T^{\prime}}\right)\right) \\
& =\sum_{T^{\prime}} \operatorname{dim}\left(X^{T^{\prime}}\right)=\sum_{T^{\prime}} \operatorname{dim}\left(V^{T^{\prime}}\right)=\operatorname{dim}(V) .
\end{aligned}
$$

Thus, $\pi$ is surjective. Let $d$ be its degree, and let $d_{T^{\prime}}$ be the degree of $\pi_{T^{\prime}}$. Then we have by Lemma 16

$$
e_{x} X=d e_{0} V=d \prod_{T^{\prime}} e_{0}\left(V^{T^{\prime}}\right)=\frac{d}{\prod_{T^{\prime}} d_{T^{\prime}}} \prod_{T^{\prime}} e_{x}\left(X^{T^{\prime}}\right) .
$$

If moreover each $X^{T^{\prime}}$ is smooth, then we can take each $\pi_{T^{\prime}}$ to be the identity. So each $d_{T^{\prime}}$ is 1 , and $c=d$ is an integer.

(ii) $\Rightarrow$ (iii) Because $e_{x} X$ is homogeneous of degree $-\operatorname{dim}(X)$, we obtain $\operatorname{dim}(X)=\sum_{T^{\prime}} \operatorname{dim}\left(X^{T^{\prime}}\right)$

(iii) $\Rightarrow$ (i) Because $\mathbb{P}(X)$ is projective and rationally smooth, the spectral sequence associated with the fibration $\mathbb{P}(X) \times_{T} E_{T} \rightarrow B_{T}$ degenerates (by the criterion of Deligne, see e.g. 35]). Thus, the $S$-module $H_{T}^{*}(\mathbb{P}(X))$ is free, and the map

$$
\rho: H_{T}^{*}(\mathbb{P}(X)) /\left(S_{+}\right) \rightarrow H^{*}(\mathbb{P}(X))
$$

is an isomorphism.

On the other hand, $\mathbb{P}(X)^{T}$ has no odd cohomology because it is the disjoint union of the $\mathbb{P}\left(X^{T^{\prime}}\right)$, and each $X^{T^{\prime}}$ is rationally smooth. Thus, $\mathbb{P}(X)^{T}$ has no odd equivariant cohomology as well. By the localization theorem, the odd equivariant cohomology of $\mathbb{P}(X)$ vanishes; thus, the cohomology of $\mathbb{P}(X)$ is concentrated in even degree.

Moreover, we have

$$
\begin{aligned}
\chi(\mathbb{P}(X))=\operatorname{rk}_{S} H_{T}^{*}(\mathbb{P}(X))=\operatorname{rk}_{S} H_{T}^{*}\left(\mathbb{P}(X)^{T}\right)=\chi\left(\mathbb{P}(X)^{T}\right) \\
=\sum_{T^{\prime}} \chi\left(\mathbb{P}\left(X^{T^{\prime}}\right)\right)=\sum_{T^{\prime}} \operatorname{dim}\left(X^{T^{\prime}}\right)=\operatorname{dim}(X) .
\end{aligned}
$$

Thus, $\mathbb{P}(X)$ has the rational cohomology of projective space of dimension $\operatorname{dim}(X)-1$. 
Example (Schubert varieties, continued)

Let $w, x$ in $W$ such that $x \leq w$. Notation being as in the previous section, rational smoothness of $X(w)$ at $x$ is equivalent to $P_{y, w}=1$ for all $y \in W$ such that $x \leq y \leq w$, where $P_{y, w}$ denotes the Kazhdan-Lusztig polynomial [37 Theorem A2 (this can be checked as in the beginning of the proof of Theorem 18).

Set

$$
\Phi(x, w):=\left\{\alpha \in x\left(\Phi^{+}\right) \mid s_{\alpha} x \leq w\right\} .
$$

Observe that $\Phi(x, w)$ contains $\Phi^{-} \cap x\left(\Phi^{+}\right)$(which is the set of all $\alpha \in x\left(\Phi^{+}\right)$ such that $\left.s_{\alpha} x \leq x\right)$, with complement the set of all $\alpha \in x\left(\Phi^{+}\right)$such that $x<s_{\alpha} x \leq w$. Indeed, for any $\alpha \in \Phi$, we have either $s_{\alpha} x<x$ or $x<s_{\alpha} x$.

We can now state the following result, where (i) is due to A. Arabia [4], (ii) and (iii) to S. Kumar [40], and (iv) to J. Carrell and D. Peterson [16].

Corollary 19 (i) There exists a homogeneous polynomial $J(x, w) \in S_{\mathbb{Z}}$ such that

$$
e_{x} X(w)=J(x, w) \prod_{\alpha \in \Phi(x, w)} \alpha^{-1} .
$$

(ii) The point $x$ is smooth in $X(w)$ if and only if $J(x, w)=1$, that is,

$$
e_{x} X(w)=\prod_{\alpha \in \Phi(x, w)} \alpha^{-1} .
$$

(iii) The point $x$ is rationally smooth in $X(w)$ if and only if: For any $y \in W$ such that $x \leq y<w$, the polynomial $J(y, w)$ is constant, that is, there exists a positive integer $d(y, w)$ such that

$$
e_{y} X(w)=d(y, w) \prod_{\alpha \in \Phi(y, w)} \alpha^{-1} .
$$

(iv) The set of weights of $T$ in the tangent space $T_{x} X(w)$ contains $\Phi(x, w)$, and we have

$$
l(w) \leq|\Phi(x, w)| \leq \operatorname{dim} T_{x} X(w) .
$$

Moreover, $x$ is rationally smooth in $X(w)$ if and only if: For any $y \in W$ such that $x \leq y<w$, we have $l(w)=|\Phi(y, w)|$. Finally, $x$ is smooth in $X(w)$ if and only if: $x$ is rationally smooth, and $|\Phi(x, w)|=\operatorname{dim} T_{x} X(w)$. 
Proof We begin by proving (ii). Let $T^{\prime} \subset T$ be a subtorus of codimension 1. Then $X(w)_{x}^{T^{\prime}} \neq x$ if and only if $T^{\prime}=\operatorname{ker}(\alpha)$ for some $\alpha \in \Phi(x, w)$; in this case, $X(w)_{x}^{T^{\prime}}$ is a smooth curve, isomorphic to the one-dimensional $T$-module with weight $\alpha$ (see the notes of T. A. Springer in this volume, or Example 1 in Section 2). In particular, $e_{x} X(w)^{k e r(\alpha)}=\alpha^{-1}$. Thus, (ii) follows from Theorem 17.

Now we prove (i). By the argument of Theorem 17, there exists a finite $T$-equivariant morphism

$$
\pi: X(w)_{x} \rightarrow V
$$

where $V$ is a $T$-module with weight set $\Phi(x, w)$ (for a concrete construction of $\pi$, embed $X(w)_{x}$ into $x U^{-} x^{-1}$; the latter is $T$-equivariantly isomorphic to a module with weight set $x\left(\Phi^{+}\right)$, which projects onto $\left.V\right)$. Let $d$ be the degree of $\pi$, and let $Y \subset V$ be its image. Then we have $\pi_{*}\left[X(w)_{x}\right]=d[Y]$ in $A_{*}^{T}(V)$, whence $e_{x} X(w)=d e_{0} Y$. Moreover, because $V$ is a $T$-module, the $S_{\mathbb{Z}}$-module $A_{*}^{T}(V)$ is freely generated by $[V]$. Write $[Y]=J(Y)[V]$ with $J(Y) \in S_{\mathbb{Z}}$, then

$$
e_{0} Y=J(Y) e_{0} V=J(Y) \prod_{\alpha \in \Phi(x, w)} \alpha^{-1}
$$

whence (i) with $J(x, w)=d J(Y)$.

The implication $(\Leftarrow)$ in (iii) follows from Theorem 18. For $(\Rightarrow)$, we use the slice $\mathcal{N}_{x, w}$ to the orbit $B x$ in $X(w)$ whose construction was recalled in the previous section. The product map

$$
U_{x} \times \mathcal{N}_{x, w} \rightarrow X(w)
$$

is an open immersion with image $X(w)_{x}$. Because $U_{x}$ is an affine space, $x$ is rationally smooth in $X(w)$ if and only if it is in $\mathcal{N}_{x, w}$. Now we argue by induction over $l(w)-l(x)=\operatorname{dim} \mathcal{N}_{x, w}$. If this number is zero, then $x=w$ and $X(w)$ is smooth at $x$. In the general case, the induction hypothesis tells us that $X(w)$ is rationally smooth at $y$ for all $y \in W$ such that $x<y \leq w$. Because the set of rationally smooth points of $X(w)$ is open and $B$-stable, it follows that $X(w) \backslash \overline{B x}$ is rationally smooth. Thus, $\mathcal{N}_{x, w} \backslash x$ is rationally smooth as well, and Theorem 18 applies.

We already saw that $\Phi(x, w)$ is the set of weights of tangent spaces to $x$ at $T$-stable curves in $X(w)$. This proves the first assertion of (iv), and the inequality $|\Phi(x, w)| \leq \operatorname{dim} T_{x} X(w)$ as well. 
The inequality $l(w) \leq|\Phi(x, w)|$ follows from finiteness of the morphism $\pi: X(w)_{x} \rightarrow V$. Moreover, equality holds if and only if $\pi$ is surjective, or equivalently,

$$
e_{x} X(w)=d \prod_{\alpha \in \Phi(x, w)} \alpha^{-1}
$$

where $d$ is the degree of $\pi$. So the criterion for rational smoothness follows from (iii).

If $x$ is smooth in $X(w)$, then it is certainly rationally smooth, and the weights of $T$ in $T_{x} X(w)$ are the weights of $T$-stable curves through $x$ in $X(w)$, that is, the elements of $\Phi(x, w)$. For the converse, observe that

$$
\operatorname{dim} X(w)=\ell(w)=|\Phi(x, w)|=\operatorname{dim} T_{x} X(w) .
$$

Let us give some applications of these criteria. Consider first the case where $l(w)=l(x)+1$. As in Section 4 , write $w=s_{\beta} x$ where $\beta \in \Phi^{+} \cap x\left(\Phi^{+}\right)$. Then $\Phi(x, w)$ consists of $\Phi^{-} \cap x\left(\Phi^{+}\right)$and of $\beta$; moreover, $e_{x} \mathcal{N}_{x, w}=\beta^{-1}$. By (ii), it follows that $x$ is a smooth point of $X(w)$, that is, the $T$-variety $\mathcal{N}_{x, w}$ is isomorphic to the module with weight $\beta$. In other words, Schubert varieties are smooth in codimension 1 (in fact, they are known to be normal 44]).

Consider now the case where $l(w)=l(x)+2$. Then the open Bruhat interval $(x, w)$ contains exactly two elements $y_{1}, y_{2}$ (this follows from the fact that, in any closed Bruhat interval, the number of elements of even length equals the number of elements of odd length [19] Proposition 3.6). Writing $y_{j}=s_{\beta_{j}} w$ as above, we see that $\Phi(x, w)$ consists of $\Phi^{-} \cap x\left(\Phi^{+}\right)$together with $\beta_{1}$ and $\beta_{2}$. By (iv), it follows that Schubert varieties are rationally smooth in codimension 2, a result of Kazhdan and Lusztig [37].

It is easy to construct examples of Schubert varieties which are rationally smooth, but singular in codimension 2. Indeed, let $\alpha, \beta$ be distinct simple roots which are connected in the Dynkin diagram. Then we saw in Section 4 that

$$
e_{1} X\left(s_{\alpha} s_{\beta} s_{\alpha}\right)=-e_{s_{\alpha}} X\left(s_{\alpha} s_{\beta} s_{\alpha}\right)=\frac{-\left\langle\beta, \alpha^{\vee}\right\rangle}{\alpha \beta s_{\alpha}(\beta)} .
$$

So the 3 dimensional Schubert variety $X\left(s_{\alpha} s_{\beta} s_{\alpha}\right)$ is rationally smooth at the $B$-fixed point, and hence everywhere; but it is singular along the curve $X\left(s_{\alpha}\right)$ whenever $\left\langle\beta, \alpha^{\vee}\right\rangle \leq-2$.

In other words, for any non simply laced group $G$, there exist singular, rationally smooth Schubert varieties. On the other hand, by an unpublished 
result of D. Peterson, rational smoothness implies smoothness for Schubert varieties of simply laced $G$ [16]. In the case where $G=\mathrm{SL}_{n}$, this is due to Deodhar [20] and follows from (iv) together with the fact that the equality $|\Phi(x, w)|=\operatorname{dim} T_{x} X(w)$ holds there for all $x$ and $w$ 41] Theorem 1.

Further applications of (rational) smoothness criteria can be found in 16] and 40 .

\section{References}

[1] M. F. Atiyah and R. Bott: The moment map and equivariant cohomology, Topology 23 (1984), 1-28.

[2] A. Arabia: Cycles de Schubert et cohomologie équivariante de $K / T$, Invent. math. 85 (1986), 39-52.

[3] A. Arabia: Cohomologie T-équivariante de la variété des drapeaux d'un groupe de Kac-Moody, Bull. Soc. math. France 117 (1989), 129-165.

[4] A. Arabia: Classe d'Euler équivariante et points rationnellement lisses, preprint, December 1997.

[5] M. Audin: The topology of torus actions on symplectic manifolds, Birkhäuser, Boston 1991.

[6] A. Bialynicki-Birula: Some theorems on actions of algebraic groups, Ann. of Math. 98 (1973), 480-497.

[7] E. Bifet, C. De Concini and C. Procesi: Cohomology of regular embeddings, Adv. Math. 82 (1990), 1-34.

[8] A. Borel: Sur la cohomologie des espaces fibrés principaux et des espaces homogènes des groupes de Lie compacts, Ann. of Math. 57 (1953), 115-207.

[9] W. Borho, J-L. Brylinski and R. MacPherson: Nilpotent Orbits, Primitive Ideals, and Characteristic Classes, Birkhäuser, Boston 1989.

[10] R. Bott: A residue formula for holomorphic vector fields, J. Differential Geom. 1 (1967), 311-330. 
[11] N. Bourbaki: Groupes et algèbres de Lie, Chapitres 7 et 8, CCLS, Paris 1975.

[12] M. Brion: Sur l'image de l'application moment, p. 177-192 in: Séminaire d'algèbre Paul Dubreil et Marie-Paul Malliavin, Lecture Notes in Math. 1296, Springer, New York 1987.

[13] M. Brion: Piecewise polynomial functions, convex polytopes, and enumerative geometry, in: Parameter Spaces (P. Pragacz, ed.), Banach Center Publications, 1996, 25-44.

[14] M. Brion: Equivariant Chow groups for torus actions, Journal of Transformation Groups 2 (1997), 225-267.

[15] M. Brion and P. Polo: Generic singularities of certain Schubert varieties, preprint 1997.

[16] J. Carrell: The Bruhat graph of a Coxeter group, a conjecture of Deodhar, and rational smoothness of Schubert varieties, pp. 53-62 in: Algebraic groups and their generalizations (W. Haboush and B. Parshall, eds.), American Mathematical Society, Providence 1994.

[17] T. Chang and T. Skjelbred: The topological Schur lemma and related results, Ann. of Math. 100 (1974), 307-321.

[18] C. De Concini and C. Procesi: Complete symmetric varieties, pp. 144 in: Invariant Theory, Lecture Note in Math. 996, Springer-Verlag, New York 1983.

[19] V. Deodhar: Some characterizations of the Bruhat ordering on a Coxeter group and determination of the relative Möbius function, Invent. math. 39 (1977), 187-198.

[20] V. Deodhar: Local Poincaré duality and nonsingularity of Schubert varieties, Comm. Alg. 13 (1985), 1379-1388.

[21] D. Edidin and W. Graham: Characteristic classes in the Chow ring, J. Alg. Geom. 6 (1997), 431-443.

[22] D. Edidin and W. Graham: Equivariant intersection theory, Invent. math., to appear. 
[23] D. Edidin and W. Graham: Localization in equivariant intersection theory and Bott's residue formula, Amer. J. Math., to appear.

[24] W. Fulton: Intersection Theory, Springer, New York 1984.

[25] W. Fulton, R. MacPherson, F. Sottile and B. Sturmfels: Intersection theory on spherical varieties, J. Alg. Geom. 4 (1995), 181-193.

[26] H. Gillet: Riemann-Roch theorems for higher algebraic $K$-theory, Adv. Math. 40 (1981), 203-289.

[27] M. Goresky, R. Kottwitz and R. MacPherson: Equivariant cohomology, Koszul duality, and the localization theorem, Invent. math. 131 (1998), $25-84$.

[28] A. Grothendieck: Torsion homologique et sections rationnelles, dans: Séminaire Chevalley, Anneaux de Chow et applications, École Normale Supérieure 1958.

[29] V. Guillemin and S. Sternberg: Multiplicity-free spaces, J. Differ. Geom. 19 (1984), 31-56.

[30] H. Hopf: Über die Topologie der Gruppen-Mannigfaltigkeiten und ihrer Verallgemeinerungen, Ann. of Math. 42 (1941), 22-52.

[31] W. Y. Hsiang: Cohomology Theory of Topological Transformation Groups, Springer, New York 1975.

[32] D. Husemoller: Fiber Bundles, third edition, Springer-Verlag, New York 1994.

[33] P. Iglesias: Les $S O(3)$-variétés symplectiques et leur classification en dimension 4, Bull. Soc. math. France 119 (1991), 371-396.

[34] A. Joseph: On the variety of a highest weight module, J. Algebra 88 (1984), 238-278.

[35] R. Joshua: Vanishing of odd-dimensional intersection cohomology, Math. Z. 195 (1987), 239-253.

[36] K. Kawakubo: The theory of transformation groups, Oxford University Press, Oxford 1991. 
[37] D. Kazhdan and G. Lusztig: Representations of Coxeter groups and Hecke algebras, Invent. math. 53 (1979), 165-184.

[38] F. Kirwan: Cohomology of quotients in symplectic and algebraic geometry, Mathematical Notes 31, Princeton University Press, Princeton 1984.

[39] B. Kostant and S. Kumar: The nil Hecke ring and cohomology of $G / P$ for a Kac-Moody group G, Adv. Math. 62 (1986), 187-237.

[40] S. Kumar: The nil Hecke ring and singularity of Schubert varieties, Invent. math. 123 (1996), 471-506.

[41] V. Lakshmibai and C. S. Seshadri: Singular locus of a Schubert variety, Bull. Amer. Math. Soc. (N.S.) 11 (1984), 363-366.

[42] E. Lerman: Symplectic cuts, Math. Res. Lett. 2 (1995), 247-258.

[43] P. Littelmann and C. Procesi: Equivariant Cohomology of Wonderful Compactifications, p. 219-262 in: Operator Algebras, Unitary Representations, Enveloping Algebras, and Invariant Theory, Birkhäuser, Basel 1990.

[44] V. Mehta and V Srinivas: Normality of Schubert varieties, Amer. J. of Math. 109 (1987), 987-989.

[45] W. Rossmann: Equivariant multiplicities on complex varieties, pp. 313330 in: Orbites unipotentes et faisceaux pervers, Astérisque 173-174, Soc. math. France 1989.

[46] H. Sumihiro: Equivariant completion, J. Math. Kyoto Univ. 14 (1974), $1-28$.

[47] C. Woodward: The classification of transversal multiplicity-free actions, Ann. Glob. An. Geom. 14 (1996), 3-42. 